Research Article

\title{
Singularity Analysis of Composite Laminated Piezoelectric Rectangular Plate Structure with 1 : 2 Internal Resonance
}

\author{
Yuhong Guo $\mathbb{D}^{1},{ }^{1}$ Yuhua Guo, ${ }^{2,3}$ Wei Zhang, ${ }^{4}$ and Ruiping Wen $\mathbb{D I}^{1}$ \\ ${ }^{1}$ Department of Mathematics, Taiyuan Normal University, Jinzhong 030619, China \\ ${ }^{2}$ State Key Laboratory of Space-Ground Integrated Information Technology, Beijing 100095, China \\ ${ }^{3}$ Beijing Institute of Satellite Information Engineering, Beijing 100095, China \\ ${ }^{4}$ Beijing Key Laboratory on Nonlinear Vibrations and Strength of Mechanical Structures, College of Mechanical Engineering, \\ Beijing University of Technology, Beijing 100124, China
}

Correspondence should be addressed to Yuhong Guo; gst9901@163.com

Received 8 February 2021; Accepted 24 July 2021; Published 17 August 2021

Academic Editor: Gordon Huang

Copyright (C) 2021 Yuhong Guo et al. This is an open access article distributed under the Creative Commons Attribution License, which permits unrestricted use, distribution, and reproduction in any medium, provided the original work is properly cited.

This study investigates the dynamical behavior of the composite laminated piezoelectric rectangular plate with $1: 2$ internal resonance near the singularity using the extended singularity theory method. Based on the previous four-dimensional averaged equations of polar coordinates where the partial derivative terms are not equal to zero, the universal unfolding with codimension 3 of the proposed system is given. The main material parameters that affect the dynamic behavior of the laminated piezoelectric rectangular composite plate near the singularity under transverse excitation are revealed by the transition set of universal unfolding with codimension 3. In addition, the plots of the transition set in three bifurcation parameters space are discussed. These numerical results can show that the stability near the singularity of the proposed system is better when period ratio is less than zero.

\section{Introduction}

Singularity theory has been widely applied as a qualitative bifurcation analysis method. It can use the uniform and clear method to deal with various complex bifurcation problems and establish comprehensive links between system dynamic behavior and system parameters with singularity theory. But so far, the application of singularity theory in the chaotic dynamics of dynamical systems faces challenges.

The introduction of the singularity theory to the singledegree-of-freedom system with a single parameter can lead to complex and interesting bifurcation phenomena, such as the pitchfork bifurcation. Schaeffer and Golubitsky [1] investigated the bifurcation phenomenon of a model under the chemical reaction. Martinet [2] discussed the open fold of smooth mapping buds under the strong equivalence and gave various forms of open fold theorem. Chen and Langford [3] gave the C-L method, by combining the L-S method and singularity theory, and studied the bifurcation of the periodic solution for nonlinear dynamical equation undergoing parametric excitation. Jin and Zou [4] studied a restrained pipe conveying fluid and obtained dynamical behaviors by singularity theory in different persistent regions. Chen et al. [5] gave the bifurcation analysis of an arch structure with the parametric and forced excitations in codimension 5 . Nigol et al. $[6,7]$ analyzed the torsional galloping mechanism under a galloping excitation. Yu et al. [8] also studied the torsional feedback mechanism under a galloping excitation. Then, with the gradual adding of bifurcation parameters, the singularity theory has been applied to a single-degree-of-freedom system with multiparameters. Futer et al. [9] and Sitta [10] gave classification of bifurcation problems under codimension not greater than 1, considering the bifurcation parameter symmetry. Lari-Lavassani and Lu [11] gave a full and accessible account of unfolding and finite determinacy and stability theorems of the multiparameter bifurcation problem. Seyranian and Mailybaev [12] studied the multiparameter stability theory and analyzed the 
influence of system parameters on the stability. Qin and Chen [13] analyzed a bifurcation system with two parameters. Zhang and Chen [14] studied stability and bifurcation behaviors for a model of simply supported functionally graded materials rectangular plate subjected to the transversal and in-plane excitations by means of combination of analytical and numerical methods. Guo and Zhang [15] studied the bifurcation problems of the composite laminated piezoelectric rectangular plate structure with three bifurcation parameters by singularity theory in the case of $1: 2$ internal resonance, the sign function is employed to the universal unfolding of bifurcation equations in this system, the proposed approach can ensure the nondegenerate conditions of the universal unfolding of bifurcation equations in this system to be satisfied, the study presents the proposed system with three bifurcation parameters is a high codimensional bifurcation problem with codimension 4 , and 6 forms of universal unfolding are given.

The current piezoelectric materials, which include piezoelectric lead-zirconate-titanate (PZT) and piezoelectric polyvinylidene fluoride (PVDF), are new type of functional materials in engineering fields. Piezoelectric materials can be used as the actuators and sensors in engineering structures. Tauchert et al. [16] reviewed the theoretical research progress of the piezoelectric intelligent composite structure. Halim and Reza Moheimani [17] studied that piezoelectric materials were glued or embedded into controlled structures as sensing elements to achieve vibration suppression by consuming or diverting the mechanical energy of controlled structures. Zhang et al. [18] investigated the nonlinear oscillations and chaotic dynamics of a parametrically excited simply supported symmetric crossply laminated composite rectangular thin plate with the geometric nonlinearity and nonlinear damping. Della and Shu [19] reviewed various models and numerical analysis results of piezoelectric composite laminated plates. Hao et al. [20] analyzed the nonlinear oscillations, bifurcations, and chaos of a functionally graded materials plate. Karnaukhov and Tkachenko Ya [21] studied the active damping of nonstationary vibrations of a hinged rectangular plate with distributed piezoelectric actuators by the dynamic-programming method. Liu et al. [22] studied the nonlinear forced vibrations of functionally graded material (FGM) sandwich cylindrical shells with porosities on an elastic substrate and analyzed the effects of the core-to-thickness ratio, porosity volume fraction, power-law exponent, and external excitation on nonlinear forced vibration characteristics of FGM sandwich shells with porosities. Liu et al. [23] developed a new solution approach to solve nonlinear forced vibrations of functionally graded (FG) piezoelectric shells in multiphysics fields; the novel feature of this approach is that it can efficiently obtain the unstable solution and tackle the difficult problems in mathematics encountered during formulation. Zhang et al.
[24] established the governing equations of motion for the nonlinear oscillations of a simply supported symmetric crossply composite laminated piezoelectric plate subjected to the transverse, in-plane excitations, and the excitation loaded by piezoelectric layers and studied the periodic and chaotic dynamics of the composite laminated piezoelectric plate.

The singularity theory for two-degree-of-asymmetry freedom bifurcation systems with a single parameter has been well developed by Golubitsky and Schaeffer [25]. The universal unfolding of bifurcation equations was obtained, and the conclusion showed that the number of codimension was equal to the number of auxiliary parameters. Present, there is limited research about the singularity theory for two-degree-of-asymmetry freedom bifurcation systems with multiparameters. In two-degreeof asymmetry freedom bifurcation systems with multiparameters, the number of auxiliary parameters is less than codimension, which is contradiction with the previous conclusion. In this work, the sign function is introduced to solve this problem and ensure nondegenerate conditions of universal unfolding of the bifurcation equations are satisfied, and determining the summation of the number of sign functions and the number of auxiliary parameters is equal to codimension. Then, for the dynamical equations of the composite laminated piezoelectric rectangular plate structure in the case of principal parametric resonance and $1 / 2$ subharmonic resonance for the first-order mode and primary resonance for the second-order mode because only transverse nonlinear oscillations of the composite laminated piezoelectric rectangular plate are considered, the governing equations of motion can be reduced to a two-degree-freedom nonlinear system under combined parametric and external excitations by using Galerkin's method [24]. By employing sign function, the universal unfolding of the 1 : 2 internal resonance bifurcation equations is derived. The transition sets in the parameters plane are calculated, and the bifurcation diagrams are depicted. The main material parameters that affect the dynamic behavior of the laminated piezoelectric rectangular composite plate near the singularity under transverse excitation are revealed by universally unfolding with codimension 3 . The motivation of this work is to provide theoretical basis for the materials selection in the product design stage.

\section{Bifurcation Equations of the Composite Laminated Piezoelectric Rectangular Plate with $1: 2$ Internal Resonance}

The nondimensional motion equations of the laminated piezoelectric composite rectangular plate as shown in (1) are taken into consideration [24]: 


$$
\left\{\begin{array}{l}
\ddot{w}_{1}+\omega_{1}^{2} w_{1}+\mu_{1} \dot{w}_{1}+\left[\alpha_{2} \cos \left(\Omega_{1} t\right)+\alpha_{3} \cos \left(\Omega_{2} t\right)+\alpha_{4} \cos \left(\Omega_{4} t\right)\right] w_{1} \\
+\alpha_{5} w_{1}^{2} w_{2}+\alpha_{6} w_{2}^{2} w_{1}+\alpha_{7} w_{1}^{3}+\alpha_{8} w_{2}^{3}=F_{1} \cos \left(\Omega_{3} t\right) \\
\ddot{w}_{2}+\omega_{2}^{2} w_{2}+\mu_{2} \dot{w}_{2}+\left[\beta_{2} \cos \left(\Omega_{1} t\right)+\beta_{3} \cos \left(\Omega_{2} t\right)+\beta_{4} \cos \left(\Omega_{4} t\right)\right] w_{1} \\
+\beta_{5} w_{2}^{2} w_{1}+\beta_{6} w_{1}^{2} w_{2}+\beta_{7} w_{2}^{3}+\beta_{8} w_{1}^{3}=F_{2} \cos \left(\Omega_{3} t\right)
\end{array}\right.
$$

where $w_{1}$ and $w_{2}$ are the amplitudes of the first-order and second-order modes for the composite laminated piezoelectric rectangular plate vibration, $F_{1}=F_{2}=-16 q / 3 \pi^{2}$ denotes the transverse excitation, $\mu_{1}$ and $\mu_{2}$ are the structure damping coefficients, and other coefficients in (1) are presented in Appendix.

Consider the case of principal parametric resonance and $1: 2$ internal resonance. In this resonant case, there are the following resonant relations:

$$
\begin{aligned}
& \omega_{1}^{2}=\frac{1}{4} \omega^{2}+\varepsilon \sigma_{1}, \\
& \omega_{2}^{2}=\omega^{2}+\varepsilon \sigma_{2}, \\
& \Omega_{1}=\omega, \\
& \Omega_{2}=\Omega_{3} \\
& \omega_{2} \approx 2 \omega_{1},
\end{aligned}
$$

where $\omega_{1}$ and $\omega_{2}$ are the two different linear natural frequencies, and $\sigma_{1}$ and $\sigma_{2}$ are the two detuning parameters.

By using the multiple scale method [24], it can obtain the averaging equations:

$$
\left\{\begin{array}{l}
\dot{a}_{1}=-\frac{1}{2} \mu_{1} a_{1}-\frac{1}{4}\left(\alpha_{2}+\alpha_{3}+\alpha_{4}\right) a_{1} \sin 2 \phi_{1}, \\
a_{1} \dot{\phi}_{1}=\frac{1}{2} \sigma_{1} a_{1}-\frac{1}{4} \alpha_{6} a_{1} a_{2}^{2}-\frac{3}{8} \alpha_{7} a_{1}^{3}-\frac{1}{4}\left(\alpha_{2}+\alpha_{3}+\alpha_{4}\right) a_{1} \cos 2 \phi_{1}, \\
\dot{a}_{2}=-\frac{1}{2} \mu_{2} a_{2}-\frac{1}{4} F_{2} \sin \phi_{2}, \\
a_{2} \dot{\phi}_{2}=\frac{1}{4} \sigma_{2} a_{2}-\frac{1}{8} a_{1}^{2} a_{2}-\frac{3}{16} \beta_{7} a_{2}^{3}-\frac{1}{4} F_{2} \cos \phi_{2} .
\end{array}\right.
$$

Using the relations between trigonometric functions, the bifurcation equations can be determined from (3) as follows:

$$
\left\{\begin{array}{l}
\left(\dot{a}_{1}+\frac{1}{2} \mu_{1} a_{1}\right)^{2}+\left(a_{1} \dot{\phi}_{1}-\frac{1}{2} \sigma_{1} a_{1}+\frac{1}{4} \alpha_{6} a_{1} a_{2}^{2}+\frac{3}{8} \alpha_{7} a_{1}^{3}\right)^{2}=\left(\frac{1}{4}\left(\alpha_{2}+\alpha_{3}+\alpha_{4}\right) a_{1}\right)^{2} \\
\left(\dot{a}_{2}+\frac{1}{2} \mu_{2} a_{2}\right)^{2}+\left(a_{2} \dot{\phi}_{2}-\frac{1}{4} \sigma_{2} a_{2}+\frac{1}{8} a_{1}^{2} a_{2}+\frac{3}{16} \beta_{7} a_{2}^{3}\right)^{2}=\left(\frac{1}{4} F_{2}\right)^{2}
\end{array}\right.
$$

Expanding (4) leads to the following bifurcation equations:

$$
\left\{\begin{array}{l}
g_{1}=k_{11} a_{1}^{6}+k_{12} a_{1}^{4} a_{2}^{2}+k_{13} a_{1}^{2} a_{2}^{4}+k_{14} \sigma_{1} a_{1}^{4}+k_{15} \sigma_{1} a_{1}^{2} a_{2}^{2}+\sigma_{1}^{2} a_{1}^{2}+k_{16} a_{1}^{2}+\tau_{1}=0, \\
g_{2}=k_{21} a_{2}^{6}+\frac{1}{4} a_{1}^{4} a_{2}^{2}+k_{22} a_{1}^{2} a_{2}^{4}+k_{23} \sigma_{2} a_{2}^{4}-\sigma_{2} a_{1}^{2} a_{2}^{2}+\sigma_{2}^{2} a_{2}^{2}+4 \mu_{2}^{2} a_{2}^{2}-F_{2}^{2}+\tau_{2}=0,
\end{array}\right.
$$

where 


$$
\begin{aligned}
k_{11}= & \frac{9}{16} \alpha_{7}^{2}, \\
k_{12}= & \frac{3}{4} \alpha_{6} \alpha_{7}, \\
k_{13}= & \frac{1}{4} \alpha_{6}^{2}, \\
k_{14}= & -\frac{3}{2} \alpha_{7}, \\
k_{15}= & -\alpha_{6}, \\
k_{16}= & \mu_{1}^{2}-\frac{1}{4}\left(\alpha_{2}+\alpha_{3}+\alpha_{4}\right)^{2}, \\
\tau_{1}= & 4 \dot{a}_{1}\left(\dot{a}_{1}+\mu_{1} a_{1}\right)+\dot{\phi}_{1}\left[\left(4 \dot{\phi}_{1}+3 \alpha_{7} a_{1}^{2}+2 \alpha_{6} a_{2}^{2}-4 \sigma_{1}\right) a_{1}^{2}\right], \\
k_{21}= & \frac{9}{16} \beta_{7}^{2}, \\
\tau_{22}= & \frac{3}{4} \beta_{7}, \\
k_{23}= & -\frac{3}{2} \beta_{7}, \\
k_{2} & \dot{a}_{2}\left(\dot{a}_{2}+\mu_{2} a_{2}\right)+\dot{\phi}_{2}\left[\left(16 \dot{\phi}_{2}+4 a_{1}^{2}+6 \beta_{7} a_{2}^{2}-8 \sigma_{2}\right) a_{2}^{2}\right] .
\end{aligned}
$$

In (6), $\tau_{1}$ and $\tau_{2}$ refer to the combination of the gradients of displacement and phase angle. It can be referred to as the periodic parameter in (5). Studies on the dynamical behavior near the singularity have been carried out in the literature for the cases where $\tau_{1}=0$ and $\tau_{2}=0$ [15]. Therefore, when $\tau_{1} \neq 0, \tau_{2} \neq 0$, and $\sigma_{1}=\sigma_{2}$, the study on the dynamical behavior near the singularity is focused in this work.

\section{Singularity Analysis for $1: 2$ \\ Internal Resonance}

Proposition 1. The universal unfolding of (5) is given as

$$
\begin{aligned}
G & =\left(G_{1}, G_{2}\right) \\
& =\left(a_{1}^{2}+2 \varepsilon_{1} \sigma_{1} a_{2}+2 \varepsilon_{3} F_{2}+\tau_{1}, a_{2}^{2}-F_{2}^{2}+2 \varepsilon_{2} F_{2} a_{1}+\tau_{2}\right)
\end{aligned}
$$

where $\varepsilon_{i}(i=1,2,3)$ is the sign function. The proof process of Proposition 1 will be given later in this work.

When $\tau_{1} \neq 0$ and $\tau_{2} \neq 0$, (7) is further written as

$$
\begin{aligned}
G & =\left(G_{1}, G_{2}\right) \\
& =\left(a_{1}^{2}+2 \varepsilon_{1} \sigma_{1} a_{2}+2 \varepsilon_{3} F_{2}+\tau_{1}, a_{2}^{2}-F_{2}^{2}+2 \varepsilon_{2} F_{2} a_{1}+\kappa \tau_{1}\right),
\end{aligned}
$$

where $\kappa=\tau_{2} / \tau_{1}$ is called the period ratio in this work.

In the following analysis, the transition set of the universal unfolding (8) is discussed.

Equation (8) for bifurcation points is obtained as

$$
\begin{aligned}
a_{1}^{2}+2 \varepsilon_{1} \sigma_{1} a_{2}+2 \varepsilon_{3} F_{2}+\tau_{1} & =0, \\
a_{2}^{2}-F_{2}^{2}+2 \varepsilon_{2} F_{2} a_{1}+\kappa \tau_{1} & =0, \\
\frac{a_{1}}{\varepsilon_{2} F_{2}}=\frac{\varepsilon_{1} \sigma_{1}}{a_{2}} & =\frac{\varepsilon_{3}}{\varepsilon_{2} a_{1}-F_{2}} .
\end{aligned}
$$

Multiplying $-\kappa$ in equation (9a) and then substituting it into equation (9b), obtain

$$
\kappa\left(a_{1}^{2}+2 \varepsilon_{1} \sigma_{1} a_{2}+2 \varepsilon_{3} F_{2}\right)-a_{2}^{2}+F_{2}^{2}-2 \varepsilon_{2} F_{2} a_{1}=0 .
$$

By equation (9c), it can get

$$
\begin{aligned}
& a_{1}=\frac{F_{2} \pm \sqrt{F_{2}^{2}+4 \varepsilon_{2} \varepsilon_{3} F_{2}}}{2 \varepsilon_{2}}, \\
& a_{2}=\frac{-\varepsilon_{1} \sigma_{1} F_{2} \pm \varepsilon_{1} \sigma_{1} \sqrt{F_{2}^{2}+4 \varepsilon_{2} \varepsilon_{3} F_{2}}}{2 \varepsilon_{2} \varepsilon_{3}} .
\end{aligned}
$$

Substituting (11) into (10), the bifurcation occurs when

$$
\begin{gathered}
\kappa\left[\varepsilon_{2} \varepsilon_{3}\left(F_{2} \pm \sqrt{F_{2}^{2}+4 \varepsilon_{2} \varepsilon_{3} F_{2}}\right)^{2} \pm 4 \sigma_{1}^{2} \sqrt{F_{2}^{2}+4 \varepsilon_{2} \varepsilon_{3} F_{2}}+\left(8 \varepsilon_{2}-4 \sigma_{1}^{2}\right) F_{2}\right] \\
-\varepsilon_{2} \varepsilon_{3}\left(-\varepsilon_{1} \sigma_{1} F_{2} \pm \varepsilon_{1} \sigma_{1} \sqrt{F_{2}^{2}+4 \varepsilon_{2} \varepsilon_{3} F_{2}}\right)^{2} \mp 4 \varepsilon_{2} \varepsilon_{3} F_{2} \sqrt{F_{2}^{2}+4 \varepsilon_{2} \varepsilon_{3} F_{2}}=0
\end{gathered}
$$

Equation (8) for hysteresis points is obtained as

$$
\begin{array}{r}
a_{1}^{2}+2 \varepsilon_{1} \sigma_{1} a_{2}+2 \varepsilon_{3} F_{2}+\tau_{1}=0, \\
a_{2}^{2}-F_{2}^{2}+2 \varepsilon_{2} F_{2} a_{1}+\kappa \tau_{1}=0, \\
a_{1} a_{2}-\varepsilon_{1} \varepsilon_{2} \sigma_{1} F_{2}=0,
\end{array}
$$

$$
\left(v_{1}^{2}, v_{2}^{2}\right) \in \operatorname{range}(d G)
$$

where $v=\left(v_{1}, v_{2}\right)$. Now assume $\sigma_{1} \neq 0, F_{2} \neq 0$, and

$$
v=\left(a_{1}, \varepsilon_{1} \sigma_{1}\right),
$$

and it is noted that $\left(v_{1}^{2}, v_{2}^{2}\right) \in \operatorname{range}(d G)$ if and only if

$$
\left(v_{1}^{2}, v_{2}^{2}\right) \cdot\left(\varepsilon_{1} \sigma_{1}, a_{2}\right)=0 \text {. }
$$


Equation (15) implies that

$$
a_{1}^{2}+\varepsilon_{1} \sigma_{1} a_{2}=0
$$

multiplying by $a_{1}$ and substituting it into equation (13c), obtain

$$
a_{1}=-\varepsilon_{2} \sigma_{1}^{2 / 3} F_{2}^{1 / 3},
$$

and using equations (16) and (17), get

$$
a_{2}=-\varepsilon_{1} \sigma_{1}^{1 / 3} F_{2}^{2 / 3} \text {. }
$$

Substituting equations (17) and (18) into (10), the hysteresis occurs when

$$
F_{2}^{2}+\sigma_{1}^{2 / 3} F_{2}^{4 / 3}+\kappa\left(2 \varepsilon_{3} F_{2}-\sigma_{1}^{4 / 3} F_{2}^{2 / 3}\right)=0 .
$$

Equation (8) for the double limit points is

$$
\begin{gathered}
a_{1}^{2}+2 \varepsilon_{1} \sigma_{1} a_{2}+2 \varepsilon_{3} F_{2}+\tau_{1}=0, \\
a_{2}^{2}-F_{2}^{2}+2 \varepsilon_{2} F_{2} a_{1}+\kappa \tau_{1}=0,
\end{gathered}
$$

$$
\begin{aligned}
a_{1} a_{2}-\varepsilon_{1} \varepsilon_{2} \sigma_{1} F_{2} & =0, \\
\left(a_{11}, a_{21}\right) \neq\left(a_{12}, a_{22}\right), \quad \text { when } F_{2} & =\text { const. }
\end{aligned}
$$

Using (12), double limit points occur when

$$
\begin{aligned}
\sigma_{1}= & 0, \\
& \kappa\left(\varepsilon_{2} \varepsilon_{3}\left(F_{2} \pm \sqrt{F_{2}^{2}+4 \varepsilon_{2} \varepsilon_{3} F_{2}}\right)^{2}+8 \varepsilon_{2} F_{2}\right) \\
& \mp 4 \varepsilon_{2} \varepsilon_{3} F_{2} \sqrt{F_{2}^{2}+4 \varepsilon_{2} \varepsilon_{3} F_{2}}=0 .
\end{aligned}
$$

$\mathrm{B}, \mathrm{H}$, and $\mathrm{D}$ have equations as follows:

$$
\begin{aligned}
& \mathrm{B}:\left\{\kappa\left[\varepsilon_{2} \varepsilon_{3}\left(F_{2} \pm \sqrt{F_{2}^{2}+4 \varepsilon_{2} \varepsilon_{3} F_{2}}\right)^{2} \pm 4 \sigma_{1}^{2} \sqrt{F_{2}^{2}+4 \varepsilon_{2} \varepsilon_{3} F_{2}}+\left(8 \varepsilon_{2}-4 \sigma_{1}^{2}\right) F_{2}\right]\right. \\
& \left.-\varepsilon_{2} \varepsilon_{3}\left(-\varepsilon_{1} \sigma_{1} F_{2} \pm \varepsilon_{1} \sigma_{1} \sqrt{F_{2}^{2}+4 \varepsilon_{2} \varepsilon_{3} F_{2}}\right)^{2} \mp 4 \varepsilon_{2} \varepsilon_{3} F_{2} \sqrt{F_{2}^{2}+4 \varepsilon_{2} \varepsilon_{3} F_{2}}=0\right\}, \\
& \mathrm{H}:\left\{F_{2}^{2}+\sigma_{1}^{2 / 3} F_{2}^{4 / 3}+\kappa\left(2 \varepsilon_{3} F_{2}-\sigma_{1}^{4 / 3} F_{2}^{2 / 3}\right)=0\right\}, \\
& \mathrm{D}:\left\{\sigma_{1}=0, \kappa\left(\varepsilon_{2} \varepsilon_{3}\left(F_{2} \pm \sqrt{F_{2}^{2}+4 \varepsilon_{2} \varepsilon_{3} F_{2}}\right)^{2}+8 \varepsilon_{2} F_{2}\right) \mp 4 \varepsilon_{2} \varepsilon_{3} F_{2} \sqrt{F_{2}^{2}+4 \varepsilon_{2} \varepsilon_{3} F_{2}}=0\right\} .
\end{aligned}
$$

From the above analysis, (22a)-(22c) represent the relationship among transverse excitation $F_{2}$, period ratio $\kappa$, and detuning parameter $\sigma_{1}$ when the composite laminated piezoelectric rectangular plate structure subjected to small perturbations exists the bifurcation, hysteresis, and double limit points.

\section{Numerical Results}

The system is simulated by using MAPLE. It take amplitudes $a_{1} \geq 0, a_{2} \geq 0$, and transverse excitation $F_{2} \geq 0$. Three-dimensional sketch of transition variety of (8) is obtained by applying (22a)-(22c), in Figure 1, where B implies the bifurcation set, $\mathrm{H}$ implies the hysteresis set, and $\mathrm{D}$ implies the double limit points. From Figure 1, it can see that the whole parametric space is divided into three kinds of different persistent regions by $(22 \mathrm{a})-(22 \mathrm{c})$. It is observed that the amplitudes $a_{1}$ and $a_{2}$ have a solution in region (1), corresponding to the original system frequency response equations that have only one solution, the composite laminated piezoelectric rectangular plate structure subjected to small perturbations is in stable state. Having multiple solutions in region (2), corresponding to the multiple solutions band of the original system frequency response equations, the composite laminated piezoelectric rectangular plate structure subjected to small perturbations is in stable state. Having nonsolution in the region (3), corresponding to the original system frequency response equations have no solution, the composite laminated piezoelectric rectangular plate structure with small perturbations is in stable state. However, entering region (1) from region (2), jumping will occur, and the hysteresis will occur when entering region (2) from region (3); either way, the composite laminated piezoelectric rectangular plate structure subjected to small perturbations is in unstable state.

The bifurcation diagrams of the three kinds of different persistent regions are given in Table 1 . From Table 1 , it is observed that when the period ratio $\kappa$ changes in region (1), there is a steady solution, whereas when the period ratio $\kappa$ translates into region (2), there are multiple solutions. Furthermore, it is observed that when the period ratio $\kappa$ changes in region (3), there is no periodic solution because the amplitude $a_{1}$ is negative.

In Figure 2, it is observed that entering region (1) from region (2) causes jumping. Furthermore, it is observed that hysteresis occurs while entering region (2) from region (3). From Figure 2, it is further found that the system is stable and the motion is periodic whether period ratio $\kappa$ is chosen 


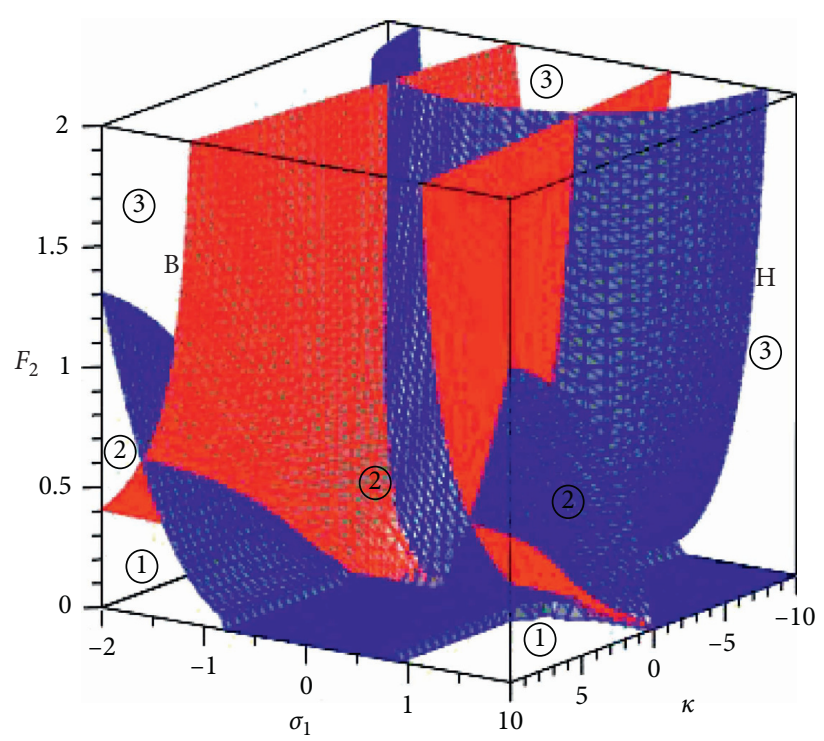

FIGURE 1: Three-dimensional sketch of transition variety of (8) when $\varepsilon_{1}=1, \varepsilon_{2}=1$, and $\varepsilon_{3}=1$, B represents the bifurcation set, and $\mathrm{H}$ represents the hysteresis set.

TABLE 1: Case of amplitude $a_{1}$ in three kinds of different persistent regions.

(1)

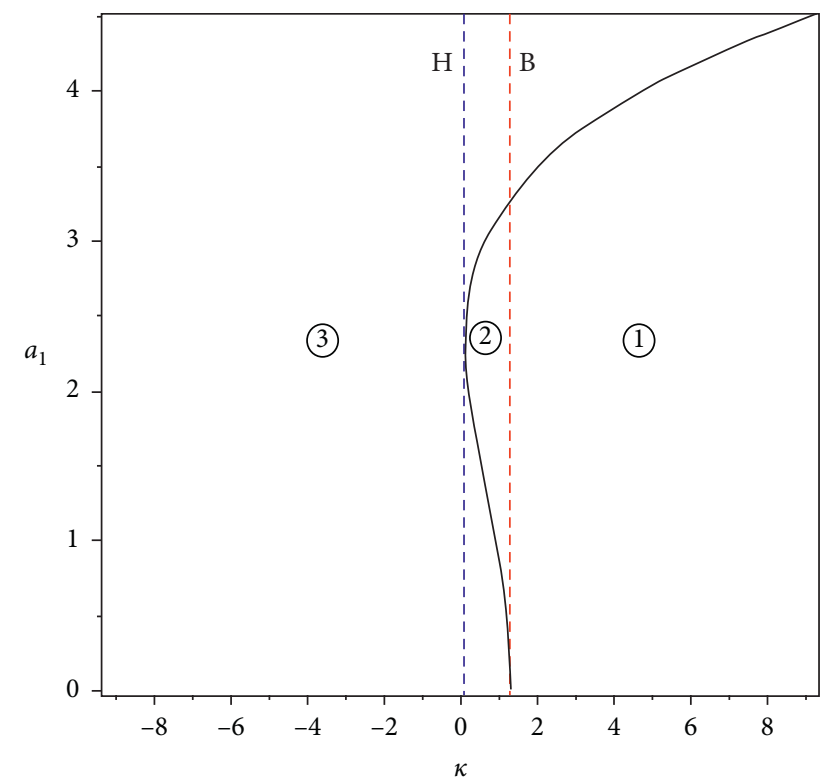

Figure 2: Plane sketch of amplitude $a_{1}$ when $\varepsilon_{1}=1, \varepsilon_{2}=1, \varepsilon_{3}=1$, $\tau_{1}=-6, F_{2}=0.2$, and $\sigma_{1}=-1$. B represents the bifurcation set, and $\mathrm{H}$ represents the hysteresis. in region (1) or (2). However, the amplitudes of the periodic motions are not the same for different initial values. Furthermore, it is observed that the system is unstable when period ratio $\kappa$ is chosen in regions (1) and (2), or regions (1), (2), and (3), or regions (2) and (3).

Case 1. The period ratio $\kappa$ is chosen in regions (1) and (2) as shown in Figure 3. Entering region (1) from region (2) causes jumping in the motion state of amplitude $a_{1}$; thus, the system is unstable and bifurcation occurs. Due to the periodicity of the period ratio $\kappa$, the case of the value of the amplitude $a_{1}$ is given as $\ldots \longrightarrow$ single value $\longrightarrow$ more values $\longrightarrow$ single value $\longrightarrow \cdots$.

Case 2. The period ratio $\kappa$ is chosen in regions (1), (2), and (3) as shown in Figure 4. Entering region (1) from region (2), jumping will occur, the hysteresis will occur when entering region (2) from region (3), so jumping and the hysteresis will occur in the motion state of amplitude $a_{1}$; thus, the system is unstable and bifurcation occurs. Due to the periodicity of the period ratio $\kappa$, the case of the value of the amplitude $a_{1}$ is 


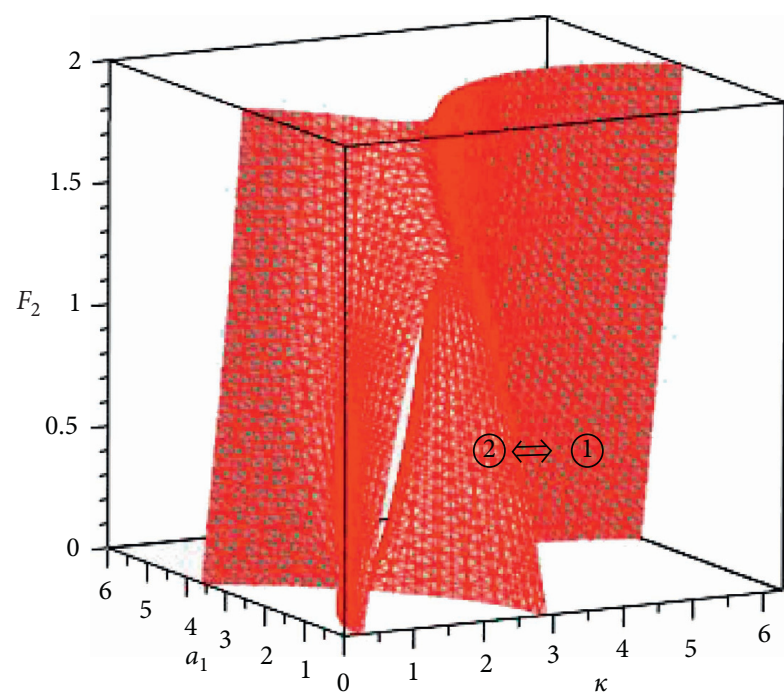

FIGURE 3: Three-dimensional sketch of amplitude $a_{1}$ when $\kappa$ is chosen in the regions (1) and (2).

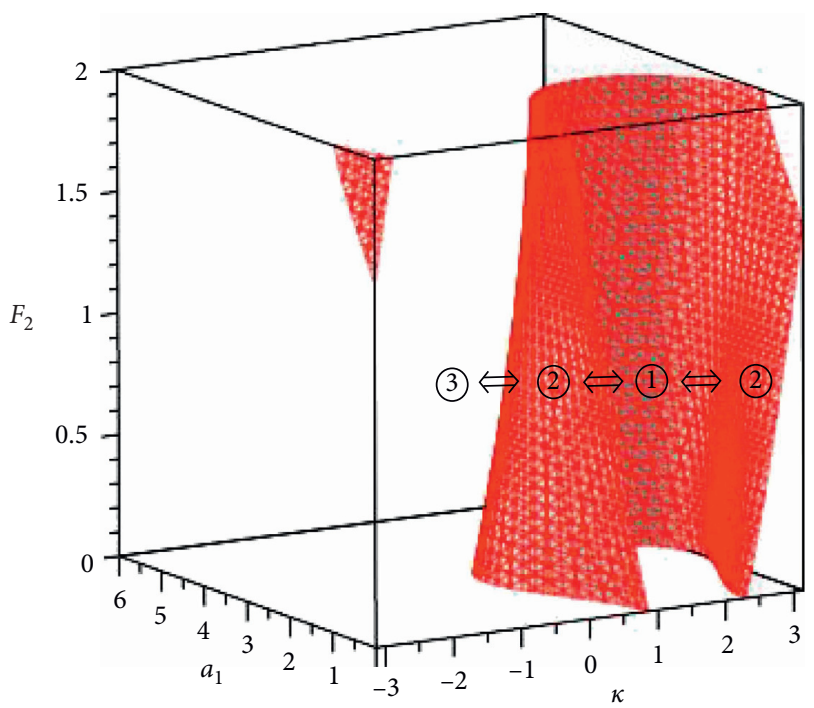

FIgURE 4: Three-dimensional sketch of amplitude $a_{1}$ when $\kappa$ is chosen in the regions (1), (2), and (3).

given as $\ldots \longrightarrow$ single value $\longrightarrow$ more values $\longrightarrow$ no solution $\longrightarrow$ more values $\longrightarrow$ single value $\longrightarrow \cdots$.

From Figure 5, it is observed that when the excitation $F_{2}$ changes, (8) is unable to compute the analytic solutions for amplitude $a_{1}$. This is due to the uncertainty of the time point from region (3) to region (2). Therefore, (8) may produce chaotic solutions.

Case 3. The period ratio $\kappa$ is chosen in regions (2) and (3) as shown in Figure 6. Entering region (2) from region (3), the hysteresis will occur in the motion state of the amplitude $a_{1}$; thus, the system is unstable and bifurcation occurs. Due to the periodicity of period ratio $\kappa$, the case of the value of the amplitude $a_{1}$ is given as ...no solution $\longrightarrow$ more values $\longrightarrow$ no solution $\longrightarrow \cdots$.

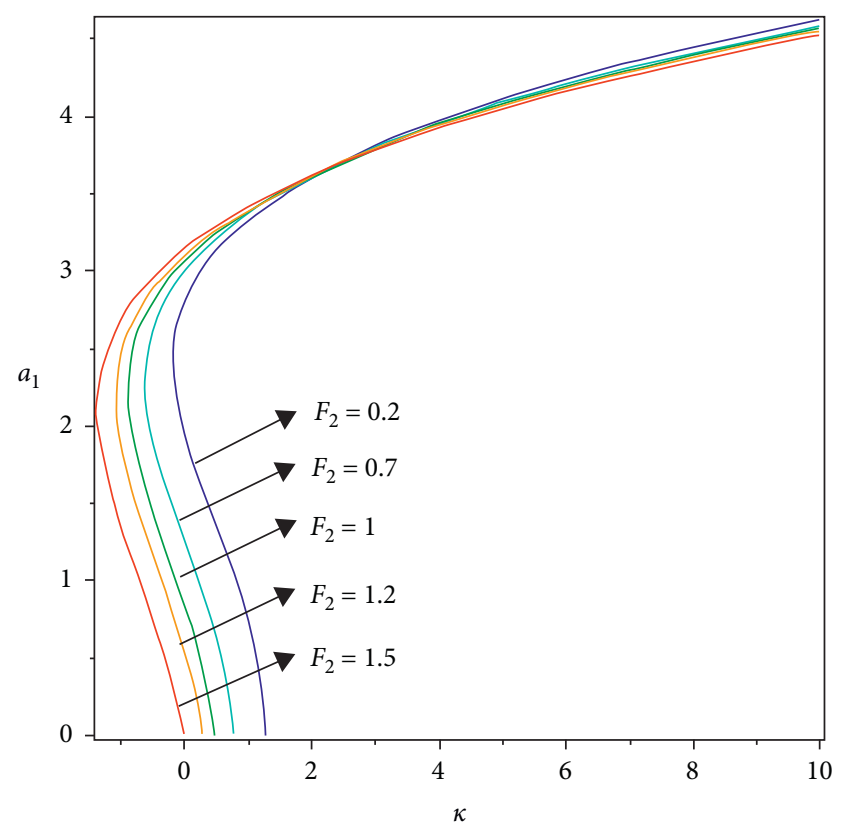

FIGURE 5: Plane sketch of amplitude $a_{1}$ when. $\tau_{1}=-6$.

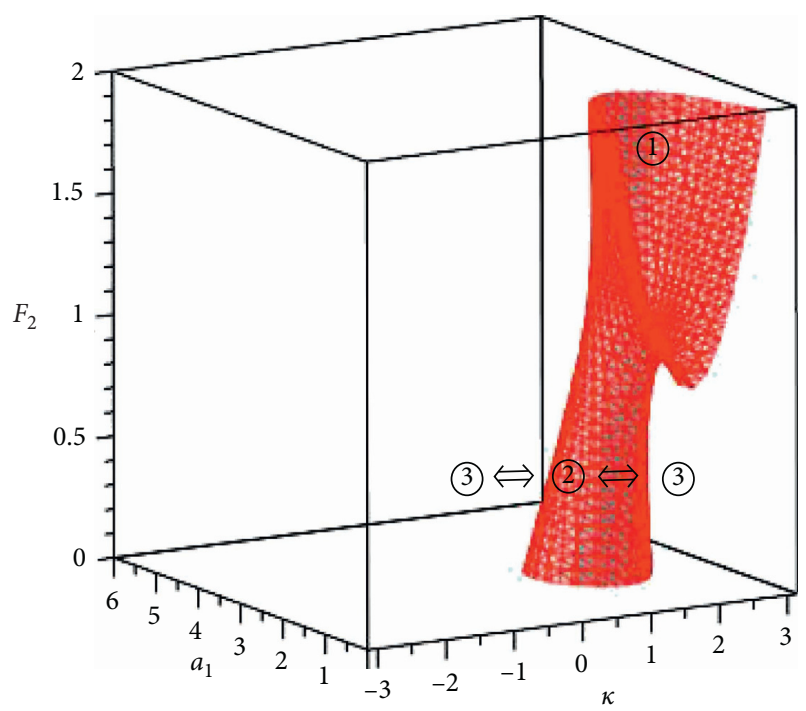

FIgURE 6: Three-dimensional sketch of amplitude $a_{1}$ when $\kappa$ is chosen in the regions (2) and (3).

It is observed that when the excitation $F_{2}$ changes, (8) may produce chaotic solutions. This is due to the uncertainty of the time point from region (3) to region (2).

In (6), the expressions of $\tau_{1}$ and $\tau_{2}$ indicate that the initial value of parameters $\alpha_{6}, \alpha_{7}$, and $\beta_{7}$ have an important influence on the range of value of period ratio $\kappa$. In other words, in addition to the transverse excitation $F_{2}$ in (8), the initial values of parameters $\alpha_{6}, \alpha_{7}$, and $\beta_{7}$ can change the range of value of period ratio $\kappa$. From the above analysis, a set of appropriate initial values for parameters $\alpha_{6}, \alpha_{7}$, and $\beta_{7}$ can be chosen, so that the period ratio $\kappa$ can be chosen in region (1) or (2); thus, the system is stable and the motion is periodic. 
By the changing of bifurcation parameters $F_{2}$ and $\sigma_{1}$, understand bifurcation properties of the composite laminated piezoelectric rectangular plate structure subjected to small perturbations. Figure 7 is the bifurcation diagrams of (9a) and (9b) when parameters $F_{2}$ and $\kappa$ change and the other parameters are fixed. In Figure $7(\mathrm{a})$, the parameters are chosen as $F_{2}=0.4, \kappa=0.3$, and $\tau_{1}=-6$, in which the bifurcation and hysteresis occur. In Figure $7(\mathrm{~b})$, the parameters are chosen as $F_{2}=0.4, \kappa=-0.3$, and $\tau_{1}=-6$, in which the bifurcation and hysteresis cannot occur. In Figure $7(\mathrm{c})$, the parameters are chosen as $F_{2}=0.5, \kappa=0.3$, and $\tau_{1}=-6$, in which the bifurcation and hysteresis occur. In Figure $7(d)$, the parameters are chosen as $F_{2}=0.5$, $\kappa=-0.3$, and $\tau_{1}=-6$, in which the bifurcation and hysteresis cannot occur. In Figure $7(\mathrm{e})$, the parameters are chosen as $F_{2}=1.9, \kappa=0.3$, and $\tau_{1}=-6$, in which the bifurcation and hysteresis occur. In Figure $7(\mathrm{f})$, the parameters are chosen as $F_{2}=1.9, \kappa=-0.3$, and $\tau_{1}=-6$, in which the bifurcation and hysteresis cannot occur. Figure 8 is also the bifurcation diagrams of (9a) and (9b) when the parameters $\sigma_{1}$ and $\kappa$ change and the other parameters are fixed. In Figure $8(\mathrm{a})$, the parameters are chosen as $\sigma_{1}=0.5, \kappa=0.3$, and $\tau_{1}=-6$, in which the bifurcation and hysteresis occur. In Figure 8(b), the parameters are chosen as $\sigma_{1}=0.5, \kappa=-0.3$, and $\tau_{1}=-6$, in which the bifurcation and hysteresis cannot occur. In Figure $8(\mathrm{c})$, the parameters are chosen as $\sigma_{1}=1, \kappa=0.3$, and $\tau_{1}=-6$, in which the bifurcation and hysteresis occur. In Figure $8(\mathrm{~d})$, the parameters are chosen as $\sigma_{1}=1$, $\kappa=-0.3$, and $\tau_{1}=-6$, in which the bifurcation and hysteresis cannot occur. In Figure $8(\mathrm{e})$, the parameters are chosen as $\sigma_{1}=2, \kappa=0.3$, and $\tau_{1}=-6$, in which the bifurcation and hysteresis occur. In Figure 8(f), the parameters are chosen as $\sigma_{1}=2, \kappa=-0.3$, and $\tau_{1}=-6$, in which the bifurcation and hysteresis cannot occur. It is observed that the jumping phenomenon and hilltop bifurcation occur at the critical point from region (1) to region (2).

Meanwhile, by choosing different bifurcation parameters $F_{2}$ and $\sigma_{1}$, displacement portraits of the amplitude $a_{1}$ are simulated. Figure 9 is displacement portraits of the amplitude $a_{1}$ of (9a) and (9b) when the parameter $F_{2}$ change and the other parameters are fixed. In Figure 9(a), the parameters are chosen as $F_{2}=0.1$ and $\sigma_{1}=1$, in which the jump phenomenon cannot occur. In Figure 9(b), the parameters are chosen as $F_{2}=0.5$ and $\sigma_{1}=1$, in which the bifurcation and hysteresis occur. In Figure $9(\mathrm{c})$, the parameters are chosen as $F_{2}=1.5$ and $\sigma_{1}=1$, in which the bifurcation and hysteresis occur. In Figure $9(\mathrm{~d})$, the parameters are chosen as $F_{2}=2$ and $\sigma_{1}=1$, in which the bifurcation and hysteresis cannot occur. Figure 10 is also displacement portraits of the amplitude $a_{1}$ of (9a) and (9b) when the parameter $\sigma_{1}$ change and the other parameters are fixed. In Figure 10(a), the parameters are chosen as $F_{2}=0.5$ and $\sigma_{1}=0.2$, in which the jump phenomenon occurs. In Figure $8(\mathrm{~b})$, the parameters are chosen as $F_{2}=0.5$ and $\sigma_{1}=0.6$, in which the jump phenomenon occurs. In Figure $8(\mathrm{c})$, the parameters are chosen as $F_{2}=0.5$ and $\sigma_{1}=1.2$, in which the jump phenomenon occurs. In Figure $8(\mathrm{~d})$, the parameters are chosen as $F_{2}=0.5$ and $\sigma_{1}=1.4$, in which the jump phenomenon cannot occur.

\section{The Proof of Proposition 1}

The proof process of Proposition 1 is briefly described as follows. The proof process is divided into five steps. The first step is to calculate the restricted tangent space as per equation (5). The second step is to obtain a strong equivalent normal form of equation (5) using the restricted tangent space. The third step is to establish the necessary and sufficient conditions that should be satisfied by the universal unfolding of the normal form. The fourth step is to calculate the polynomial space of the normal form. Finally, the fifth step is to prove the universal unfolding of the normal form.

Step 1. Calculating of the restricted tangent space of (5).

Proposition 2. The restricted tangent space $\mathrm{RT}(g, 1)$ of the germ $g(z, \lambda)$ is given as

$$
\mathrm{RT}(g, 1)=\left(\mathrm{M}^{2}+\mathrm{M}\left\langle\sigma_{1}, F_{2}\right\rangle+\left\langle F_{2}^{2}\right\rangle+\left\langle\tau_{1}\right\rangle+\left\langle\tau_{2}\right\rangle\right) \overrightarrow{\mathrm{E}}_{z, \lambda}
$$

Proof. According to Proposition 1.4 (page 169 in Golubitsky and Schaeffer II, 1985), RT $(g, 1)$ is generated (as module over $\mathrm{E}_{z, \lambda}$ ) by the sixteen mappings:

$$
\begin{aligned}
& \left(g_{1}, 0\right), \\
& \left(g_{2}, 0\right), \\
& \left(0, g_{1}\right), \\
& \left(0, g_{2}\right), \\
& a_{1}\left(g_{1, a_{1}}, g_{2, a_{1}}\right), \\
& a_{2}\left(g_{1, a_{1}}, g_{2, a_{1}}\right), \\
& \sigma_{1}\left(g_{1, a_{1}}, g_{2, a_{1}}\right), \\
& F_{2}\left(g_{1, a_{1}}, g_{2, a_{1}}\right), \\
& \tau_{1}\left(g_{1, a_{1}}, g_{2, a_{1}}\right), \\
& \tau_{2}\left(g_{1, a_{1}}, g_{2, a_{1}}\right), \\
& a_{1}\left(g_{1, a_{2}}, g_{2, a_{2}}\right), \\
& a_{2}\left(g_{1, a_{2}}, g_{2, a_{2}}\right), \\
& \sigma_{1}\left(g_{1, a_{2}}, g_{2, a_{2}}\right), \\
& F_{2}\left(g_{1, a_{2}}, g_{2, a_{2}}\right), \\
& \tau_{1}\left(g_{1, a_{2}}, g_{2, a_{2}}\right), \\
& \tau_{2}\left(g_{1, a_{2}}, g_{2, a_{2}}\right) .
\end{aligned}
$$

$\left(\mathrm{M}^{2}+\mathrm{M}\left\langle\sigma_{1}, F_{2}\right\rangle+\left\langle F_{2}^{2}\right\rangle+\left\langle\tau_{1}\right\rangle+\left\langle\tau_{2}\right\rangle\right) \overrightarrow{\mathrm{E}}_{z, \lambda}$ is generated by the following twenty mappings: 


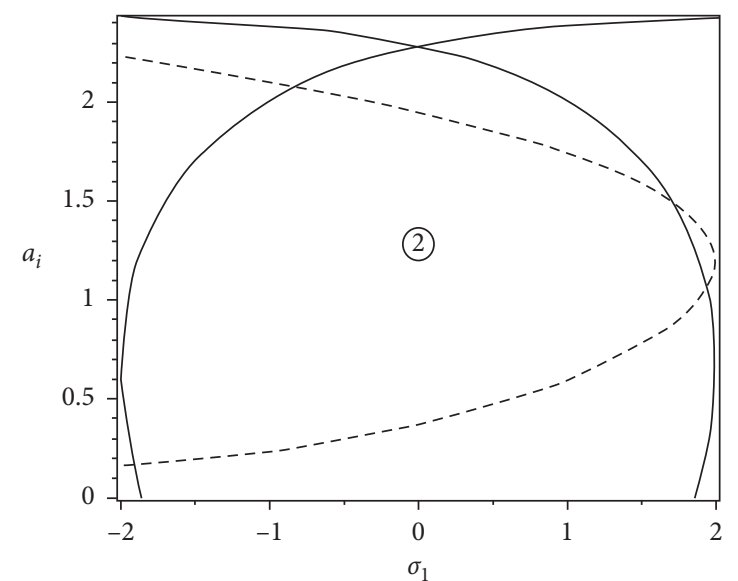

(a)

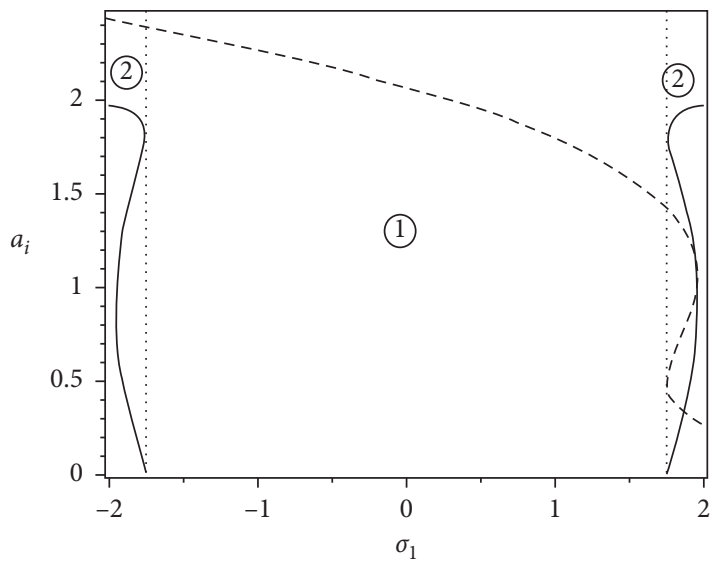

(c)

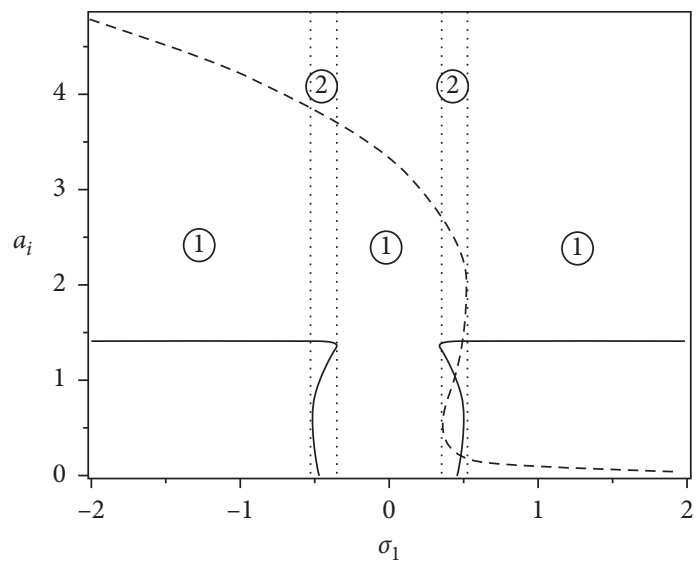

(e)

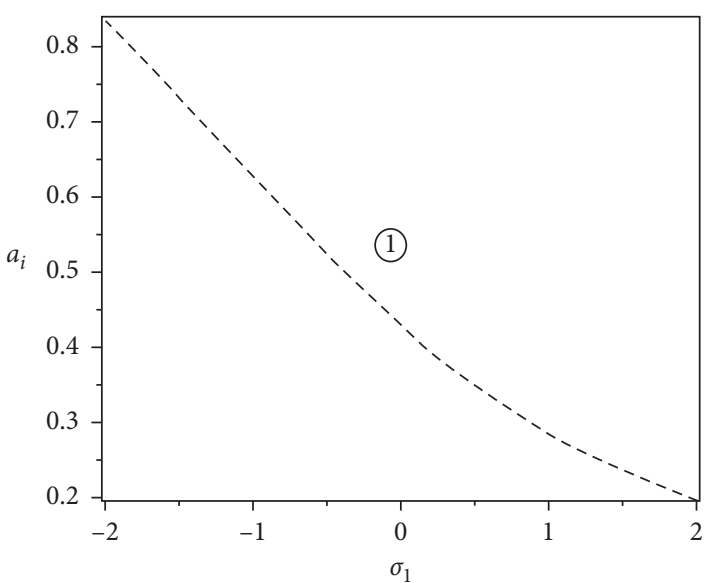

(b)

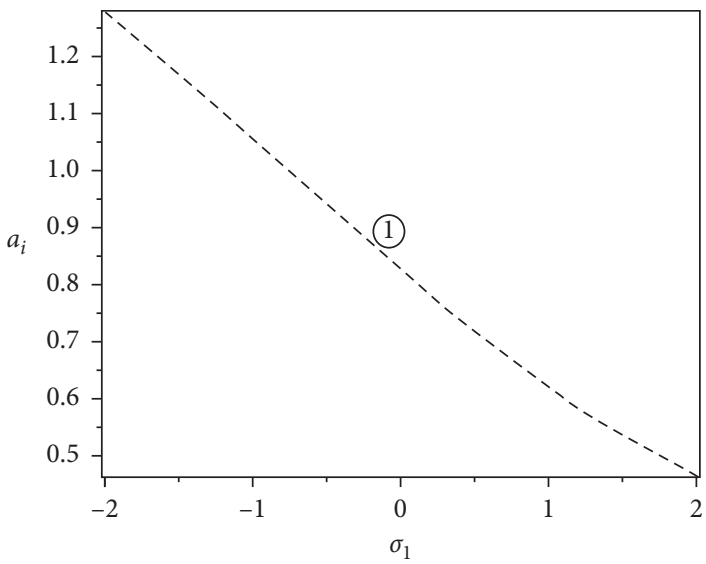

(d)

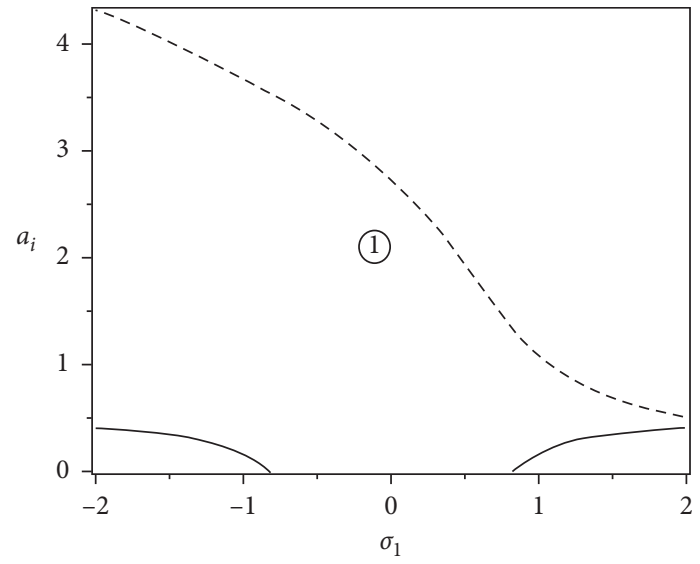

(f)

FIgURE 7: The bifurcation diagrams of $(9 \mathrm{a})$ and $(9 \mathrm{~b})$ when $\varepsilon_{1}=1, \varepsilon_{2}=1$, and $\varepsilon_{3}=1$ (left, $\kappa=0.3$; right, $\kappa=-0.3$ ). 


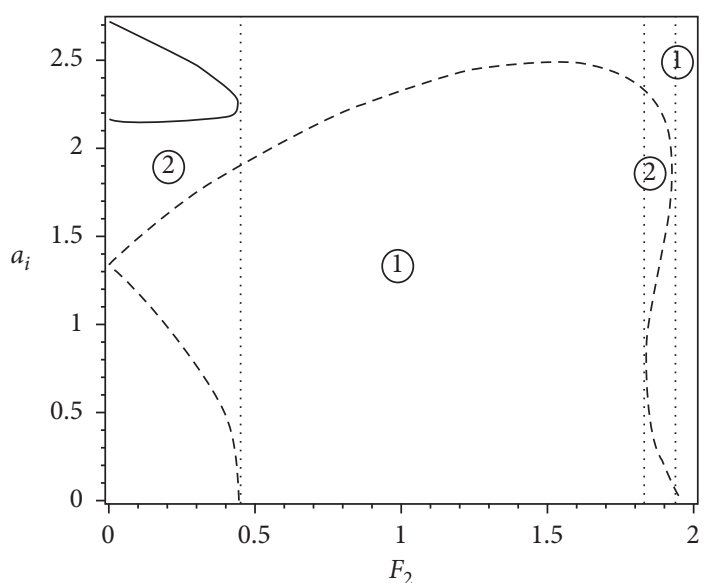

(a)

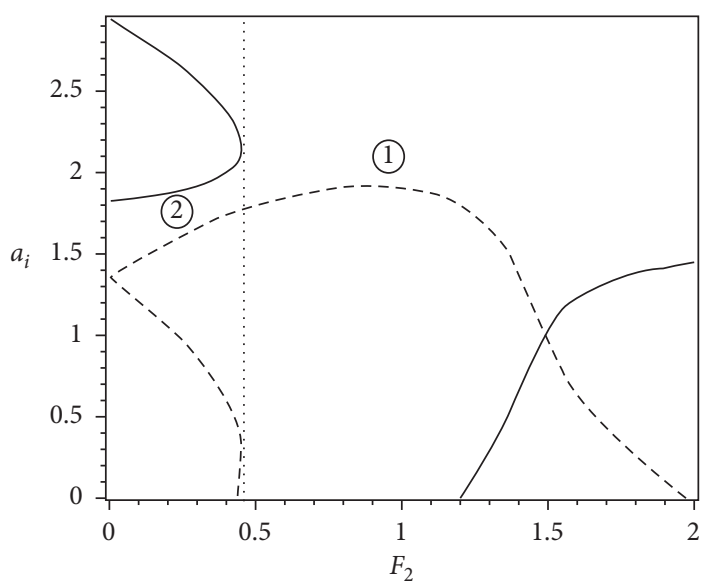

(c)

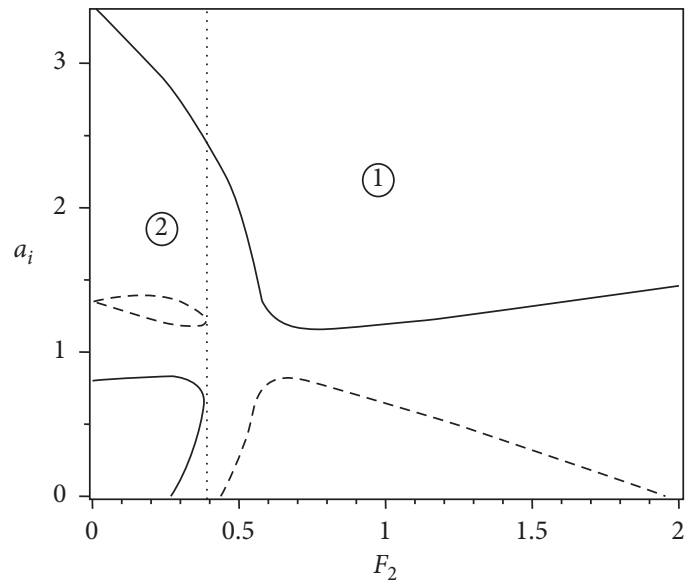

(e)

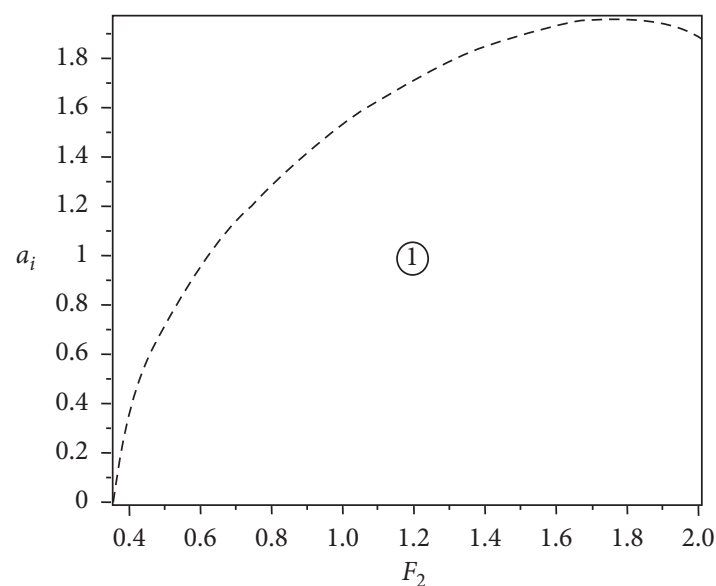

(b)

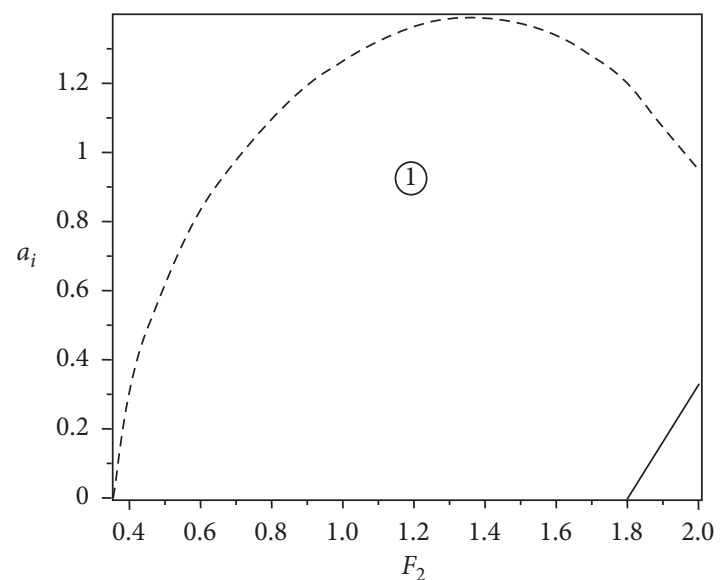

(d)

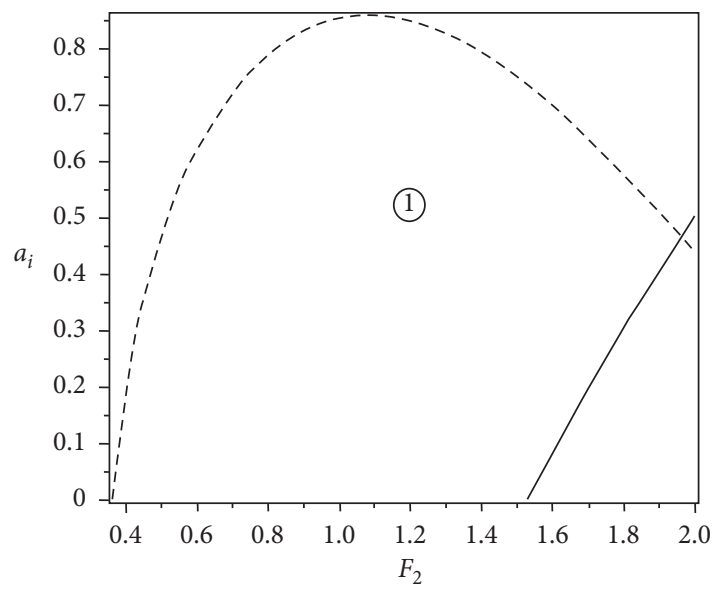

(f)

Figure 8: The bifurcation diagrams of (9a) and (9b) when $\varepsilon_{1}=1, \varepsilon_{2}=1$, and $\varepsilon_{3}=1$ (left, $\kappa=0.3$; right, $\kappa=-0.3$ ). 


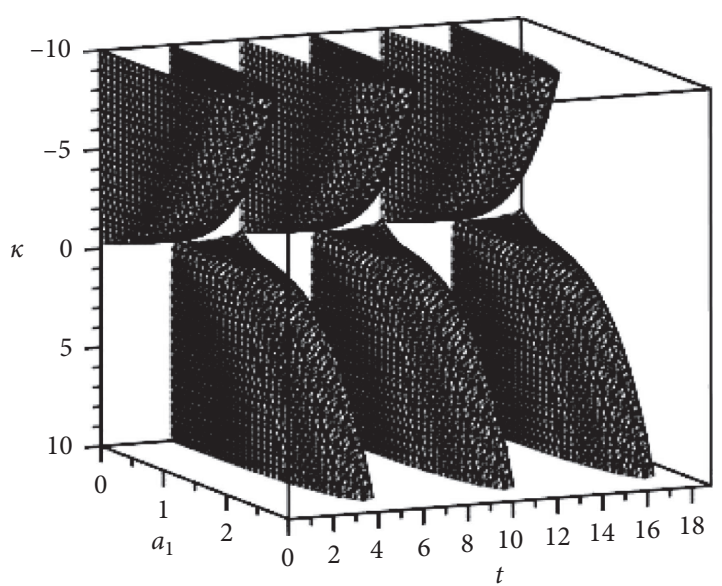

(a)

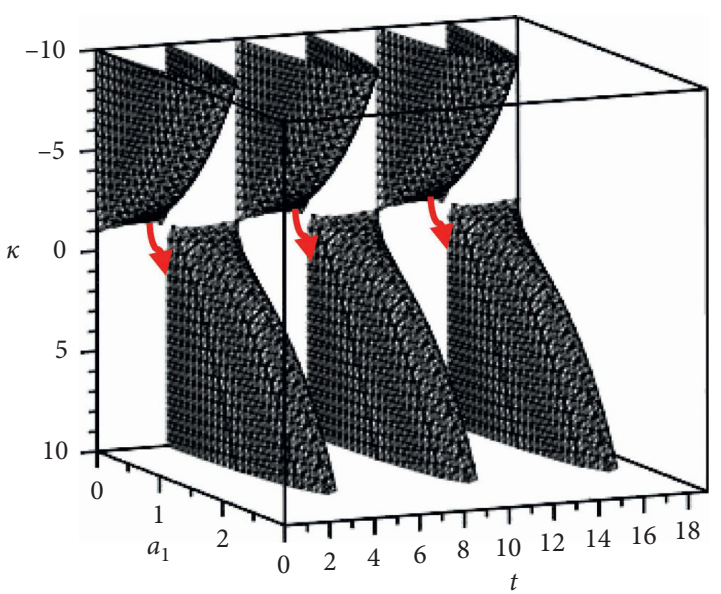

(c)

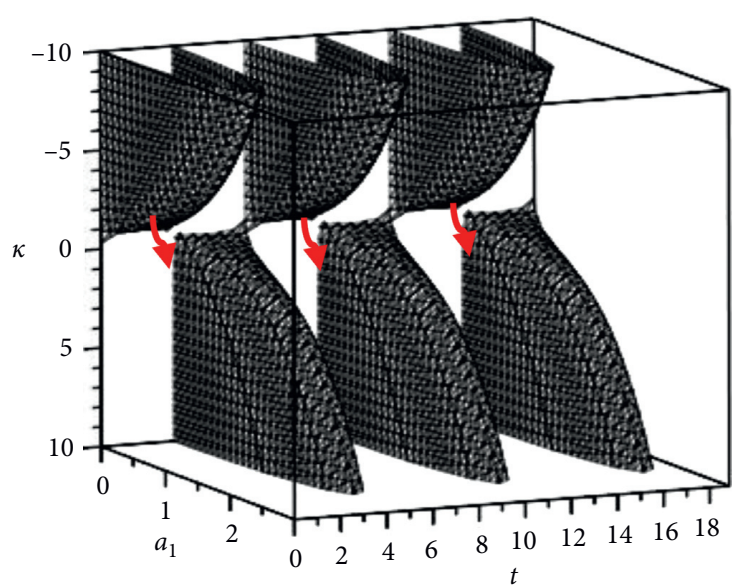

(b)

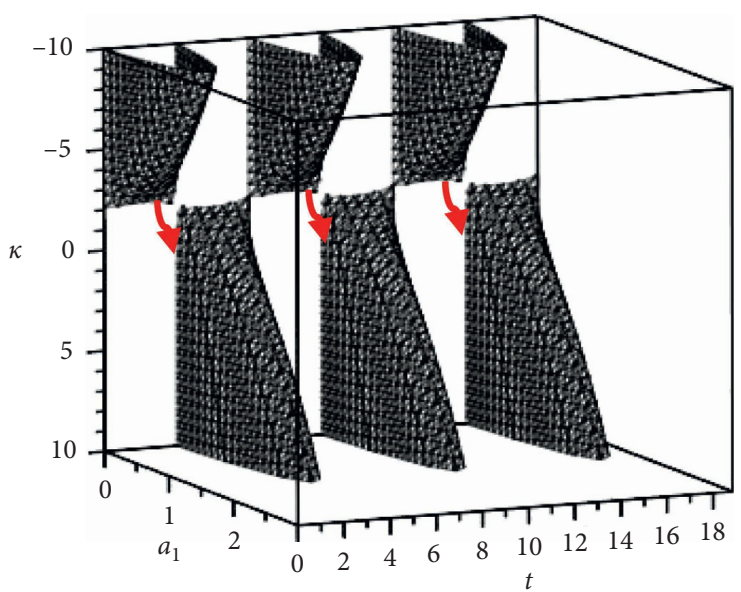

(d)

Figure 9: Displacement portraits of the amplitude $a_{1}$ of (9a) and (9b) when $\varepsilon_{1}=1, \varepsilon_{2}=1$, and $\varepsilon_{3}=1$.

$$
\begin{array}{r}
\left(a_{1}^{2}, 0\right), \\
\left(a_{1} a_{2}, 0\right), \\
\left(a_{2}^{2}, 0\right), \\
\left(\sigma_{1} a_{1}, 0\right), \\
\left(\sigma_{1} a_{2}, 0\right), \\
\left(F_{2} a_{1}, 0\right), \\
\left(F_{2} a_{2}, 0\right), \\
\left(\tau_{1}, 0\right), \\
\left(\tau_{2}, 0\right), \\
\left(F_{2}^{2}, 0\right), \\
\left(0, a_{1}^{2}\right), \\
\left(0, a_{1} a_{2}\right), \\
\left(0, a_{2}^{2}\right),
\end{array}
$$

$\left(0, \sigma_{1} a_{1}\right)$

$\left(0, \sigma_{1} a_{2}\right)$,

$\left(0, F_{2} a_{1}\right)$,

$\left(0, F_{2} a_{2}\right)$,

$\left(0, \tau_{1}\right)$,

$\left(0, \tau_{2}\right)$,

$\left(0, F_{2}^{2}\right)$.

There exists a reversible matrix $A$ between (24) and (25) when $\left(a_{1}, a_{2}, \sigma_{1}, F_{2}\right)=(0,0,0,0)$ :

$$
\left(\begin{array}{l}
u_{1} \\
u_{2}
\end{array}\right)=A\left(\begin{array}{l}
v_{1} \\
v_{2}
\end{array}\right)=\left(\begin{array}{ll}
A_{1} & A_{2} \\
A_{3} & A_{4}
\end{array}\right)\left(\begin{array}{l}
v_{1} \\
v_{2}
\end{array}\right)
$$

where 


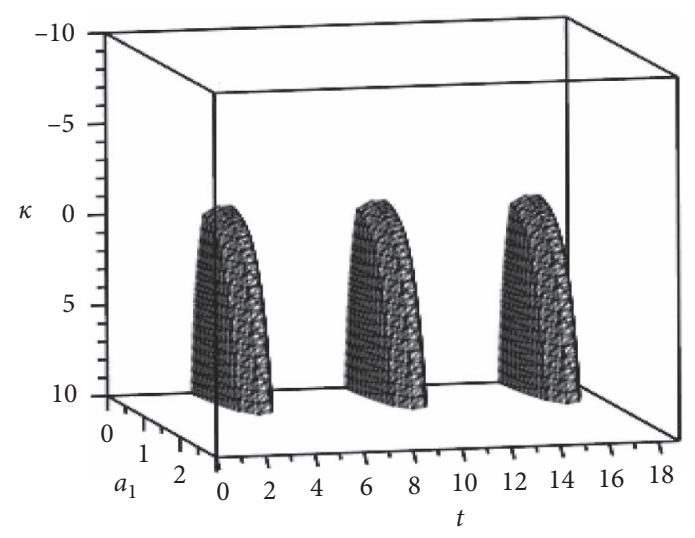

(a)

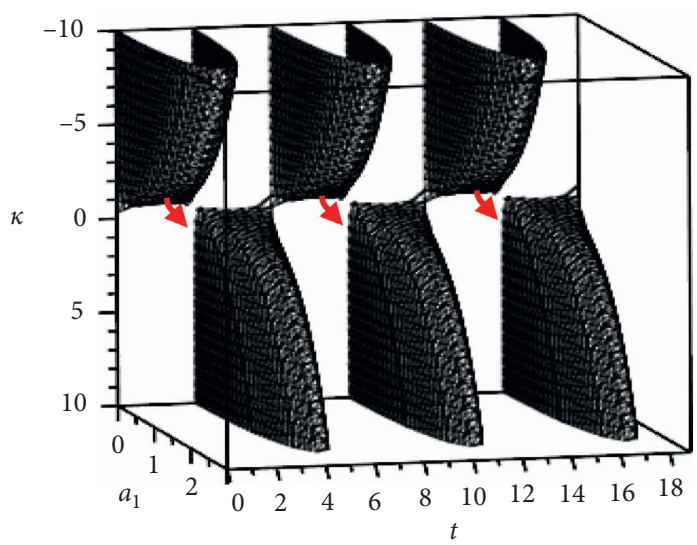

(c)

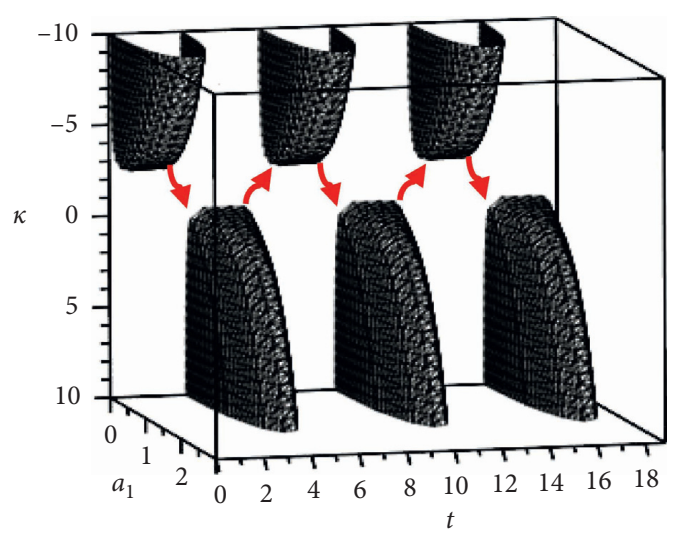

(b)

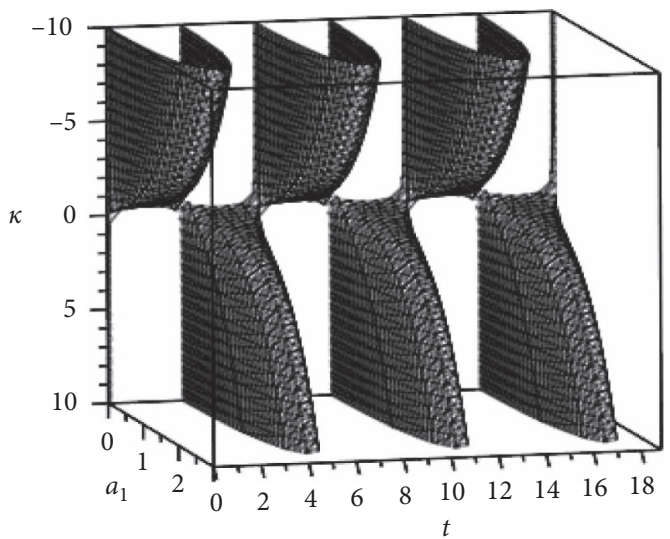

(d)

Figure 10: Displacement portraits of the amplitude $a_{1}$ of (9a) and (9b) when $\varepsilon_{1}=1, \varepsilon_{2}=1$, and $\varepsilon_{3}=1$.

$$
\begin{aligned}
& u_{1}=\left\{\left(g_{1}, 0\right) \quad\left(g_{2}, 0\right) \quad\left(0, g_{1}\right) \quad\left(0, g_{2}\right) \quad a_{1}\left(g_{1, a_{1}}, g_{2, a_{1}}\right)\right. \\
& \left.a_{2}\left(g_{1, a_{1}}, g_{2, a_{1}}\right) \quad \sigma_{1}\left(g_{1, a_{1}}, g_{2, a_{1}}\right) \quad F_{2}\left(g_{1, a_{1}}, g_{2, a_{1}}\right)\right\}_{1 \times 8}^{\mathrm{T}} \\
& u_{2}=\left\{\tau_{1}\left(g_{1, a_{1}}, g_{2, a_{1}}\right) \quad \tau_{2}\left(g_{1, a_{1}}, g_{2, a_{1}}\right) \quad a_{1}\left(g_{1, a_{2}}, g_{2, a_{2}}\right) \quad a_{2}\left(g_{1, a_{2}}, g_{2, a_{2}}\right)\right. \\
& \left.\sigma_{1}\left(g_{1, a_{2}}, g_{2, a_{2}}\right) \quad F_{2}\left(g_{1, a_{2}}, g_{2, a_{2}}\right) \quad \tau_{1}\left(g_{1, a_{2}}, g_{2, a_{2}}\right) \quad \tau_{2}\left(g_{1, a_{2}}, g_{2, a_{2}}\right)\right\}_{1 \times 8}^{T}, \\
& v_{1}=\left\{\left(a_{1}^{2}, 0\right) \quad\left(a_{1} a_{2}, 0\right) \quad\left(a_{2}^{2}, 0\right) \quad\left(\sigma_{1} a_{1}, 0\right) \quad\left(\sigma_{1} a_{2}, 0\right)\right. \\
& \left.\left(F_{2} a_{1}, 0\right) \quad\left(F_{2} a_{2}, 0\right) \quad\left(F_{2}^{2}, 0\right) \quad\left(\tau_{1}, 0\right) \quad\left(\tau_{2}, 0\right)\right\}_{1 \times 10}^{\mathrm{T}} \text {, } \\
& v_{2}=\left\{\left(0, a_{1}^{2}\right) \quad\left(0, a_{1} a_{2}\right) \quad\left(0, a_{2}^{2}\right) \quad\left(0, \sigma_{1} a_{1}\right) \quad\left(0, \sigma_{1} a_{2}\right)\right.
\end{aligned}
$$

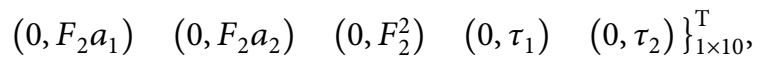

$$
\begin{aligned}
& g_{1, a_{1}}=\left[6 k_{11} a_{1}^{4}+4 k_{12} a_{1}^{2} a_{2}^{2}+2 k_{13} a_{2}^{4}+4 k_{14} \sigma_{1} a_{1}^{2}+2 k_{15} \sigma_{1} a_{2}^{2}+2\left(\sigma_{1}^{2}+k_{16}\right)\right] a_{1}, \\
& g_{2, a_{1}}=\left(a_{1}^{2} a_{2}+2 k_{22} a_{2}^{3}-2 \sigma_{1} a_{2}\right) a_{1} a_{2} \\
& g_{1, a_{2}}=\left(2 k_{12} a_{1}^{3}+4 k_{13} a_{1} a_{2}^{2}+2 k_{15} \sigma_{1} a_{1}\right) a_{1} a_{2}, \\
& g_{2, a_{2}}=\left[6 k_{21} a_{2}^{4}+\frac{1}{2} a_{1}^{4}+4 k_{22} a_{1}^{2} a_{2}^{2}+4 k_{23} \sigma_{1} a_{2}^{2}-2 \sigma_{1} a_{1}^{2}+2\left(\sigma_{1}^{2}+4 \mu_{2}^{2}\right)\right] a_{2},
\end{aligned}
$$




$$
\begin{aligned}
& A_{1}=\left(\begin{array}{cccccccccc}
c_{1} & 0 & 0 & c_{2} & 0 & 0 & 0 & 0 & 1 & 0 \\
0 & 0 & c_{3} & 0 & c_{4} & 0 & 0 & -1 & 0 & 1 \\
0 & 0 & 0 & 0 & 0 & 0 & 0 & 0 & 0 & 0 \\
0 & 0 & 0 & 0 & 0 & 0 & 0 & 0 & 0 & 0 \\
c_{5} & 0 & 0 & 0 & 0 & 0 & 0 & 0 & 0 & 0 \\
0 & c_{5} & 0 & 0 & 0 & 0 & 0 & 0 & 0 & 0 \\
0 & 0 & 0 & c_{5} & 0 & 0 & 0 & 0 & 0 & 0 \\
0 & 0 & 0 & 0 & 0 & c_{5} & 0 & 0 & 0 & 0
\end{array}\right), \\
& A_{2}=\left(\begin{array}{cccccccccc}
0 & 0 & 0 & 0 & 0 & 0 & 0 & 0 & 0 & 0 \\
0 & 0 & 0 & 0 & 0 & 0 & 0 & 0 & 0 & 0 \\
c_{1} & 0 & 0 & c_{2} & 0 & 0 & 0 & 0 & 1 & 0 \\
0 & 0 & c_{3} & 0 & c_{4} & 0 & 0 & -1 & 0 & 1 \\
c_{6} & 0 & 0 & 0 & 0 & 0 & 0 & 0 & 0 & 0 \\
0 & c_{6} & 0 & 0 & 0 & 0 & 0 & 0 & 0 & 0 \\
0 & 0 & 0 & c_{6} & 0 & 0 & 0 & 0 & 0 & 0 \\
0 & 0 & 0 & 0 & 0 & c_{6} & 0 & 0 & 0 & 0
\end{array}\right), \\
& A_{3}=\left(\begin{array}{cccccccccc}
0 & 0 & 0 & 0 & 0 & 0 & 0 & 0 & c_{5} a_{1} & 0 \\
0 & 0 & 0 & 0 & 0 & 0 & 0 & 0 & 0 & c_{5} a_{1} \\
0 & c_{7} & 0 & 0 & 0 & 0 & 0 & 0 & 0 & 0 \\
0 & 0 & c_{7} & 0 & 0 & 0 & 0 & 0 & 0 & 0 \\
0 & 0 & 0 & 0 & c_{7} & 0 & 0 & 0 & 0 & 0 \\
0 & 0 & 0 & 0 & 0 & 0 & c_{7} & 0 & 0 & 0 \\
0 & 0 & 0 & 0 & 0 & 0 & 0 & 0 & c_{7} a_{2} & 0 \\
0 & 0 & 0 & 0 & 0 & 0 & 0 & 0 & 0 & c_{7} a_{2}
\end{array}\right), \\
& A_{4}=\left(\begin{array}{cccccccccc}
0 & 0 & 0 & 0 & 0 & 0 & 0 & 0 & c_{6} a_{1} & 0 \\
0 & 0 & 0 & 0 & 0 & 0 & 0 & 0 & 0 & c_{6} a_{1} \\
0 & c_{8} & 0 & 0 & 0 & 0 & 0 & 0 & 0 & 0 \\
0 & 0 & c_{8} & 0 & 0 & 0 & 0 & 0 & 0 & 0 \\
0 & 0 & 0 & 0 & c_{8} & 0 & 0 & 0 & 0 & 0 \\
0 & 0 & 0 & 0 & 0 & 0 & c_{8} & 0 & 0 & 0 \\
0 & 0 & 0 & 0 & 0 & 0 & 0 & 0 & c_{8} a_{2} & 0 \\
0 & 0 & 0 & 0 & 0 & 0 & 0 & 0 & 0 & c_{8} a_{2}
\end{array}\right), \\
& c_{1}=k_{11} a_{1}^{4}+k_{12} a_{1}^{2} a_{2}^{2}+k_{13} a_{2}^{4}+k_{16} \text {, } \\
& c_{2}=k_{14} a_{1}^{3}+k_{15} a_{1} a_{2}^{2}+\sigma_{1} a_{1}, \\
& c_{3}=k_{21} a_{2}^{4}+\frac{1}{4} a_{1}^{4}+k_{22} a_{1}^{2} a_{2}^{2}+4 \mu_{2}^{2}, \\
& c_{4}=k_{23} a_{2}^{3}-a_{1}^{2} a_{2}+\sigma_{2} a_{2} \\
& c_{5}=6 k_{11} a_{1}^{4}+4 k_{12} a_{1}^{2} a_{2}^{2}+2 k_{13} a_{2}^{4}+4 k_{14} \sigma_{1} a_{1}^{2}+2 k_{15} \sigma_{1} a_{2}^{2}+2\left(\sigma_{1}^{2}+k_{16}\right) \text {, } \\
& c_{6}=\left(a_{1}^{2} a_{2}+2 k_{22} a_{2}^{3}-2 \sigma_{2} a_{2}\right) a_{2} \text {, } \\
& c_{7}=\left(2 k_{12} a_{1}^{3}+4 k_{13} a_{1} a_{2}^{2}+2 k_{15} \sigma_{1} a_{1}\right) a_{1},
\end{aligned}
$$




$$
c_{8}=6 k_{21} a_{2}^{4}+\frac{1}{2} a_{1}^{4}+4 k_{22} a_{1}^{2} a_{2}^{2}+4 k_{23} \sigma_{2} a_{2}^{2}-2 \sigma_{2} a_{1}^{2}+2\left(\sigma_{2}^{2}+4 \mu_{2}^{2}\right) .
$$

In (5), if $k_{16}=0$, bifurcation analysis is meaningless, so $k_{16}>0$ will be studied in this study; similarly, $k_{16}<0$ can also be analyzed. When $a_{1}=a_{2}=\sigma_{1}=F_{2}=0$, substituting $\mu_{2}>0$ into (29a)-(29h), it can obtain

$$
\begin{aligned}
& c_{1}=k_{16} \neq 0, \\
& c_{2}=0, \\
& c_{3}=4 \mu_{2}^{2}>0, \\
& c_{4}=0, \\
& c_{5}=2 k_{16} \neq 0, \\
& c_{6}=0, \\
& c_{7}=0, \\
& c_{8}=8 \mu_{2}^{2}>0 .
\end{aligned}
$$

This matrix $A$ equals

$$
\left(\begin{array}{ll}
A_{1}^{\prime} & A_{2}^{\prime} \\
A_{3}^{\prime} & A_{4}^{\prime}
\end{array}\right)
$$

where

$$
A_{1}=\left(\begin{array}{cccccccccc}
k_{16} & 0 & 0 & 0 & 0 & 0 & 0 & 0 & 1 & 0 \\
0 & 0 & 4 \mu_{2}^{2} & 0 & 0 & 0 & 0 & -1 & 0 & 1 \\
0 & 0 & 0 & 0 & 0 & 0 & 0 & 0 & 0 & 0 \\
0 & 0 & 0 & 0 & 0 & 0 & 0 & 0 & 0 & 0 \\
2 k_{16} & 0 & 0 & 0 & 0 & 0 & 0 & 0 & 0 & 0 \\
0 & 2 k_{16} & 0 & 0 & 0 & 0 & 0 & 0 & 0 & 0 \\
0 & 0 & 0 & 2 k_{16} & 0 & 0 & 0 & 0 & 0 & 0 \\
0 & 0 & 0 & 0 & 0 & 2 k_{16} & 0 & 0 & 0 & 0
\end{array}\right),
$$

$$
\begin{aligned}
A_{2} & =\left(\begin{array}{cccccccccc}
0 & 0 & 0 & 0 & 0 & 0 & 0 & 0 & 0 & 0 \\
0 & 0 & 0 & 0 & 0 & 0 & 0 & 0 & 0 & 0 \\
k_{16} & 0 & 0 & 0 & 0 & 0 & 0 & 0 & 1 & 0 \\
0 & 0 & 4 \mu_{2}^{2} & 0 & 0 & 0 & 0 & -1 & 0 & 1 \\
0 & 0 & 0 & 0 & 0 & 0 & 0 & 0 & 0 & 0 \\
0 & 0 & 0 & 0 & 0 & 0 & 0 & 0 & 0 & 0 \\
0 & 0 & 0 & 0 & 0 & 0 & 0 & 0 & 0 & 0 \\
0 & 0 & 0 & 0 & 0 & 0 & 0 & 0 & 0 & 0
\end{array}\right), \\
A_{3} & =\left(\begin{array}{llllllllll}
0 & 0 & 0 & 0 & 0 & 0 & 0 & 0 & 0 & 0 \\
0 & 0 & 0 & 0 & 0 & 0 & 0 & 0 & 0 & 0 \\
0 & 0 & 0 & 0 & 0 & 0 & 0 & 0 & 0 & 0 \\
0 & 0 & 0 & 0 & 0 & 0 & 0 & 0 & 0 & 0 \\
0 & 0 & 0 & 0 & 0 & 0 & 0 & 0 & 0 & 0 \\
0 & 0 & 0 & 0 & 0 & 0 & 0 & 0 & 0 & 0 \\
0 & 0 & 0 & 0 & 0 & 0 & 0 & 0 & 0 & 0 \\
0 & 0 & 0 & 0 & 0 & 0 & 0 & 0 & 0 & 0
\end{array}\right),
\end{aligned}
$$

$$
A_{4}=\left(\begin{array}{cccccccccc}
0 & 0 & 0 & 0 & 0 & 0 & 0 & 0 & 0 & 0 \\
0 & 0 & 0 & 0 & 0 & 0 & 0 & 0 & 0 & 0 \\
0 & 8 \mu_{2}^{2} & 0 & 0 & 0 & 0 & 0 & 0 & 0 & 0 \\
0 & 0 & 8 \mu_{2}^{2} & 0 & 0 & 0 & 0 & 0 & 0 & 0 \\
0 & 0 & 0 & 0 & 8 \mu_{2}^{2} & 0 & 0 & 0 & 0 & 0 \\
0 & 0 & 0 & 0 & 0 & 0 & 8 \mu_{2}^{2} & 0 & 0 & 0 \\
0 & 0 & 0 & 0 & 0 & 0 & 0 & 0 & 0 & 0 \\
0 & 0 & 0 & 0 & 0 & 0 & 0 & 0 & 0 & 0
\end{array}\right) .
$$

It can be seen that there are the columns with the linear correlation in the $16 \times 20$ matrix $A$, namely, the 3 th, 8 th, and 10th columns, the 11th and 19th columns, and the 13th, 
18 th, and 20 th columns. The 9 th, 10 th, 15 th, and 16 th rows and the 5 th, 7 th, 14 th, and 16 th columns are identically zero. Therefore, the 9th, 10th, 15th, and 16th rows and the 5th, 7 th, 8th, 10th, 14th, 16th, 18th, and 20th columns can be eliminated. Thus, the original $16 \times 20$ matrix has rank 12 . The proof is completed.

Step 2. The strong equivalent normal form of (5).
Proposition 3. Let

$$
g(z, \lambda)=h(z, \lambda)+q(z, \lambda)
$$

where

$$
\begin{aligned}
& h=\left(k_{16} a_{1}^{2}+\tau_{1}, 4 \mu_{2}^{2} a_{2}^{2}-F_{2}^{2}+\tau_{2}\right), \\
& q=\left(k_{11} a_{1}^{6}+k_{12} a_{1}^{4} a_{2}^{2}+k_{13} a_{1}^{2} a_{2}^{4}+k_{14} \sigma_{1} a_{1}^{4}+k_{15} \sigma_{1} a_{1}^{2} a_{2}^{2}, k_{21} a_{2}^{6}+\frac{1}{4} a_{1}^{4} a_{2}^{2}+k_{22} a_{1}^{2} a_{2}^{4}+k_{23} \sigma_{1} a_{2}^{4}-\sigma_{1} a_{1}^{2} a_{2}^{2}\right) .
\end{aligned}
$$

$\rightarrow$ If $q \in\left(\mathrm{M}^{3}+\mathrm{M}^{2}\left\langle\sigma_{1}, F_{2}\right\rangle+\mathrm{M}\left\langle F_{2}^{2}\right\rangle+\mathrm{M}\left\langle\tau_{1}\right\rangle+\mathrm{M}\left\langle\tau_{2}\right\rangle\right)$ $\overrightarrow{\mathrm{E}}_{z, \lambda}$ is a higher-order term, then $\mathrm{g}$ is strongly equivalent to $h$.

Proof. By Proposition 2, a higher order term of $\mathrm{M}^{2}+\mathrm{M}\left\langle F_{2}\right\rangle+\left\langle F_{2}^{2}\right\rangle+\left\langle\tau_{1}\right\rangle+\left\langle\tau_{2}\right\rangle$ is

$$
\mathrm{M}^{3}+\mathrm{M}^{2}\left\langle F_{2}\right\rangle+\mathrm{M}\left\langle F_{2}^{2}\right\rangle+\mathrm{M}\left\langle\tau_{1}\right\rangle+\mathrm{M}\left\langle\tau_{2}\right\rangle .
$$

For polynomial $g$, it is observed that

$$
\begin{aligned}
& \left(k_{11} a_{1}^{6}, 0\right), \\
& \left(k_{12} a_{1}^{4} a_{2}^{2}, 0\right), \\
& \left(k_{13} a_{1}^{2} a_{2}^{4}, 0\right), \\
& \left(0, k_{21} a_{2}^{6}\right), \\
& \left(0, \frac{1}{4} a_{1}^{4} a_{2}^{2}\right), \\
& \left(0, k_{22} a_{1}^{2} a_{2}^{4}\right) \text { belong to } \mathrm{M}^{3} \overrightarrow{\mathrm{E}}_{z, \lambda}, \\
& \left(k_{14} \sigma_{1} a_{1}^{4}, 0\right), \\
& \left(k_{15} \sigma_{1} a_{1}^{2} a_{2}^{2}, 0\right), \\
& \left(\sigma_{1}^{2} a_{1}^{2}, 0\right), \\
& \left(0, k_{23} \sigma_{2} a_{2}^{4}\right), \\
& \left(0, \sigma_{1} a_{1}^{2} a_{2}^{2}\right), \\
& \left(0, \sigma_{1} a_{2}^{2}\right) \text { belong to } \mathrm{M}^{2}\left\langle\sigma_{1}\right\rangle \overrightarrow{\mathrm{E}}_{z, \lambda} .
\end{aligned}
$$

Thus,

$$
q \in\left(\mathrm{M}^{3}+\mathrm{M}^{2}\left\langle\sigma_{1}, F_{2}\right\rangle+\mathrm{M}\left\langle F_{2}^{2}\right\rangle+\mathrm{M}\left\langle\tau_{1}\right\rangle+\mathrm{M}\left\langle\tau_{2}\right\rangle\right) \overrightarrow{\mathrm{E}}_{z, \lambda} .
$$

By applying Theorem 1.3 (page 168 in Golubitsky and Schaeffer II, 1985), it can get $g$ is strongly equivalent to $h$. The proof is completed.

According to Proposition 3, polynomial $g$ will be replaced by polynomial $h$ in the following discussion.

Proposition 4. The nondegenerate conditions of $h$ satisfy

$$
\begin{array}{r}
D>0, \\
\left(\tau_{1}, \tau_{2}\right) \times\left(k_{16}, 0\right) \neq 0, \\
\left(\tau_{1}, \tau_{2}\right) \times\left(0,4 \mu_{2}^{2}\right) \neq 0,
\end{array}
$$

and $h$ is equivalent to

$$
\left(a_{1}^{2}+\tau_{1}, a_{2}^{2}-F_{2}^{2}+\tau_{2}\right) .
$$

Proof. Let

$$
h(z, \lambda)=f(z)+F_{2}^{2}(0,-1)+\left(\tau_{1}, \tau_{2}\right),
$$

where $f(z)=\left(k_{16} a_{1}^{2}, 4 \mu_{2}^{2} a_{2}^{2}\right)$; according to (2.6), (2.7), and (2.8) (page 402 in Golubitsky and Schaeffer I, 1985),

$$
\begin{aligned}
Q(h) & =\left|\begin{array}{cc}
2 k_{16} a_{1} & 0 \\
0 & 8 \mu_{2}^{2} a_{2}
\end{array}\right|=16 k_{16} \mu_{2}^{2} a_{1} a_{2}, \\
D & =b^{2}-4 a c=256 k_{16}^{2} \mu_{2}^{4} .
\end{aligned}
$$

Since $k_{16} \neq 0, \mu_{2}>0$,

$$
D>0 \text {. }
$$

Applying Theorem 2.2 (page 403 in Golubitsky and Schaeffer I, 1985), choose nonzero vectors $z_{1}=(1,0)$ and $z_{2}=(0,1)$, one on each line, and let $w_{i}=f\left(z_{i}\right), i=1,2$. It can obtain

$$
\begin{aligned}
& w_{1}=\left(k_{16}, 0\right), \\
& w_{2}=\left(0,4 \mu_{2}^{2}\right) .
\end{aligned}
$$

Since $\tau_{1} \neq 0$ and $\tau_{2} \neq 0$, 


$$
\begin{aligned}
& \left(\tau_{1}, \tau_{2}\right) \times\left(k_{16}, 0\right) \neq 0, \\
& \left(\tau_{1}, \tau_{2}\right) \times\left(0,4 \mu_{2}^{2}\right) \neq 0,
\end{aligned}
$$

and it can be known that $\left(\tau_{1}, \tau_{2}\right)$ lies in quadrant 1 or quadrant 2 (page 403 in Golubitsky and Schaeffer I, 1985).

The normal form of the germ $h$ can be expressed as

$$
\left(a_{1}^{2}+\tau_{1}, a_{2}^{2}-F_{2}^{2}+\tau_{2}\right) \text {. }
$$

The proof is completed. It is observed from (45) that its universal unfolding needs complementary linear terms about the state variables and constant terms.

Step 3. The necessary and sufficient conditions that the universal unfolding of the normal form of $h$ should satisfy.

Improving Theorem 3.1 (page 409 in Golubitsky and Schaeffer I, 1985), by introducing a sign function $\varepsilon$ in (Guo and Zhang, 2019), Proposition 5 is given as follows.

\section{Proposition 5. Let}

$$
\begin{aligned}
H & \left(a_{1}, a_{2}, \sigma_{1}, F_{2}, \tau_{1}, \tau_{2}\right) \\
& =\left(h_{1}\left(a_{1}, a_{2}, \sigma_{1}, F_{2}, \tau_{1}, \tau_{2}\right), h_{2}\left(a_{1}, a_{2}, \sigma_{1}, F_{2}, \tau_{1}, \tau_{2}\right)\right),
\end{aligned}
$$

be the unfolding of a bifurcation problem $h$ in two state variables which satisfies the nondegeneracy conditions of Proposition 4. Then, $H$ is a universal unfolding of $h$ if and only if

$$
\begin{aligned}
H & \left(a_{1}, a_{2}, 0, F_{2}, \tau_{1}, \tau_{2}\right) \\
\quad & =\left(h_{1}\left(a_{1}, a_{2}, 0, F_{2}, \tau_{1}, \tau_{2}\right), h_{2}\left(a_{1}, a_{2}, 0, F_{2}, \tau_{1}, \tau_{2}\right)\right),
\end{aligned}
$$

$$
\operatorname{det}(P)=\operatorname{det}\left(\begin{array}{cccccc}
0 & h_{1, a_{1} a_{1}} & h_{1, a_{1} a_{2}} & 0 & h_{2, a_{1} a_{1}} & h_{2, a_{1} a_{2}} \\
0 & h_{1, a_{2} a_{1}} & h_{1, a_{2} a_{2}} & 0 & h_{2, a_{2} a_{1}} & h_{2, a_{2} a_{2}} \\
h_{1, \sigma_{1}} & h_{1, \sigma_{1} a_{1}} & h_{1, \sigma_{1} a_{2}} & h_{2, \sigma_{1}} & h_{2, \sigma_{1} a_{1}} & h_{2, \sigma_{1} a_{2}} \\
h_{1, F_{2}} & h_{1, F_{2} a_{1}} & h_{1, F_{2} a_{2}} & h_{2, F_{2}} & h_{2, F_{2} a_{1}} & h_{2, F_{2} a_{2}} \\
h_{1, \tau_{1}} & h_{1, \tau_{1} a_{1}} & h_{1, \tau_{1} a_{2}} & h_{2, \tau_{1}} & h_{2, \tau_{1} a_{1}} & h_{2, \tau_{1} a_{2}} \\
h_{1, \tau_{2}} & h_{1, \tau_{2} a_{1}} & h_{1, \tau_{2} a_{2}} & h_{2, \tau_{2}} & h_{2, \tau_{2} a_{1}} & h_{2, \tau_{2} a_{2}}
\end{array}\right) \neq 0
$$

where it is evaluated at $\left(a_{1}, a_{2}, \sigma_{1}, F_{2}, \tau_{1}, \tau_{2}\right)=$ $\left(0,0,0,0, \tau_{1}, \tau_{2}\right)$.

Proof. Matrix $P$ can be expressed by six vectors:

$$
P=\left(\chi_{1}, \chi_{2}, \chi_{3}, \chi_{4}, \chi_{5}, \chi_{6}\right)^{T},
$$

in which

$$
\begin{aligned}
& \chi_{1}=\left(0, h_{1, a_{1} a_{1}}, h_{1, a_{1} a_{2}}, 0, h_{2, a_{1} a_{1}}, h_{2, a_{1} a_{2}}\right), \\
& \chi_{2}=\left(0, h_{1, a_{2} a_{1}}, h_{1, a_{2} a_{2}}, 0, h_{2, a_{2} a_{1}}, h_{2, a_{2} a_{2}}\right),
\end{aligned}
$$

$$
\begin{aligned}
& \chi_{3}=\left(h_{1, \sigma_{1}}, h_{1, \sigma_{1} a_{1}}, h_{1, \sigma_{1} a_{2}}, h_{2, \sigma_{1}}, h_{2, \sigma_{1} a_{1}}, h_{2, \sigma_{1} a_{2}}\right), \\
& \chi_{4}=\left(h_{1, F_{2}}, h_{1, F_{2} a_{1}}, h_{1, F_{2} a_{2}}, h_{2, F_{2}}, h_{2, F_{2} a_{1}}, h_{2, F_{2} a_{2}}\right), \\
& \chi_{5}=\left(h_{1, \tau_{1}}, h_{1, \tau_{1} a_{1}}, h_{1, \tau_{1} a_{2}}, h_{2, \tau_{1}}, h_{2, \tau_{1} a_{1}}, h_{2, \tau_{1} a_{2}}\right), \\
& \chi_{6}=\left(h_{1, \tau_{2}}, h_{1, \tau_{2} a_{1}}, h_{1, \tau_{2} a_{2}}, h_{2, \tau_{2}}, h_{2, \tau_{2} a_{1}}, h_{2, \tau_{2} a_{2}}\right) .
\end{aligned}
$$

Substituting (45) into (50a)-(50f),

$$
\begin{aligned}
& \chi_{1}=(0,2,0,0,0,0), \\
& \chi_{2}=(0,0,0,0,0,2), \\
& \chi_{3}=(0,0,0,0,0,0), \\
& \chi_{4}=(0,0,0,0,0,0), \\
& \chi_{5}=(1,0,0,0,0,0), \\
& \chi_{6}=(0,0,0,1,0,0) .
\end{aligned}
$$

In (51), only four vectors $\chi_{1}, \chi_{2}, \chi_{5}$, and $\chi_{6}$ are linearly independent because $H$ is the universal unfolding of $h$, and there exist two complementary linearly independent vectors. It is easy to find a complementary subspace to $h$, spanned by

$$
\begin{aligned}
& \left(\varepsilon_{1} \sigma_{1} a_{2}, 0\right), \\
& \left(0, \varepsilon_{2} F_{2} a_{1}\right),
\end{aligned}
$$

where $\varepsilon_{i}$ is a sign function, $\varepsilon_{i}=+1,0,-1(i=1,2)$.

The introduced $\varepsilon$ can satisfy (47) and (48) (Guo and Zhang, 2019). From (52), it is obtained that

$$
\begin{aligned}
H & \left(a_{1}, a_{2}, 0, F_{2}, \tau_{1}, \tau_{2}\right) \\
\quad & =\left(h_{1}\left(a_{1}, a_{2}, 0, F_{2}, \tau_{1}, \tau_{2}\right), h_{2}\left(a_{1}, a_{2}, 0, F_{2}, \tau_{1}, \tau_{2}\right)\right) .
\end{aligned}
$$

Obvious, when $\varepsilon_{i}=0$,

$$
\begin{aligned}
H & \left(a_{1}, a_{2}, F_{2}, \tau_{1}, \tau_{2}, 0\right) \\
\quad & =\left(h_{1}\left(a_{1}, a_{2}, F_{2}, \tau_{1}, \tau_{2}, 0\right), h_{2}\left(a_{1}, a_{2}, F_{2}, \tau_{1}, \tau_{2}, 0\right)\right) .
\end{aligned}
$$

Substituting (53) into (48), when $\varepsilon_{i} \neq 0$,

$$
\operatorname{det}(P) \neq 0 \text {. }
$$

Conversely, in (51), there are only four vectors $\chi_{1}, \chi_{2}, \chi_{5}$, and $\chi_{6}$, which are linearly independent because $\operatorname{det}(P) \neq 0$, and the linearly independent vectors complementary to $h$ need two. It may be adopted that

$$
\begin{aligned}
& \left(\varepsilon_{1} \sigma_{1} a_{2}, 0\right), \\
& \left(0, \varepsilon_{2} F_{2} a_{1}\right) .
\end{aligned}
$$

Thus, $\quad H=\left(a_{1}^{2}+2 \varepsilon_{1} \sigma_{1} a_{2}+\tau_{1}, a_{2}^{2}-F_{2}^{2}+2 \varepsilon_{2} F_{2} a_{1}+\tau_{2}\right)$ will be obtained. The proof is completed. 
Step 4. Calculating of the polynomial space of the normal form of $h$.

Proposition 6. The polynomial space of $h$ is given by

$$
\begin{aligned}
\mathrm{R}\{ & \left.(d h)_{z, \lambda}\left(Y_{1}\right), \ldots,(d h)_{z, \lambda}\left(Y_{m}\right), h_{\lambda}, \lambda h_{\lambda}, \lambda^{2} h_{\lambda}, \ldots\right\} \\
& =\mathrm{R}\left\{\left(a_{1}, 0\right),\left(0, a_{2}\right),\left(0, F_{2}\right),(1,0),(0,1)\right\} .
\end{aligned}
$$

Proof. To derive the above equality, it should be noted that

$$
\left(\begin{array}{l}
u_{1}^{\prime} \\
u_{2}^{\prime}
\end{array}\right)=B\left(\begin{array}{l}
v_{1}^{\prime} \\
v_{2}^{\prime}
\end{array}\right)=\left(\begin{array}{ll}
B_{1} & B_{2} \\
B_{3} & B_{4}
\end{array}\right)\left(\begin{array}{l}
v_{1}^{\prime} \\
v_{2}^{\prime}
\end{array}\right)
$$

where

$$
\begin{aligned}
& u_{1}^{\prime}=\left\{\left(h_{1, a_{1}}, 0\right)\left(h_{1, a_{2}}, 0\right)\left(h_{1, F_{2}}, 0\right)\left(h_{1, \tau_{1}}, 0\right)\left(h_{1, \tau_{2}}, 0\right)\right\}_{1 \times 5}^{\mathrm{T}}, \\
& u_{2}^{\prime}=\left\{\left(0, h_{2, a_{1}}\right)\left(0, h_{2, a_{2}}\right)\left(0, h_{2, F_{2}}\right)\left(0, h_{2, \tau_{1}}\right)\left(0, h_{2, \tau_{2}}\right)\right\}_{1 \times 5}^{\mathrm{T}}, \\
& v_{1}^{\prime}=\left\{\left(a_{1}, 0\right)\left(a_{2}, 0\right)\left(F_{2}, 0\right) \quad(1,0) \quad(1,0)\right\}_{1 \times 5}^{\mathrm{T}},
\end{aligned}
$$

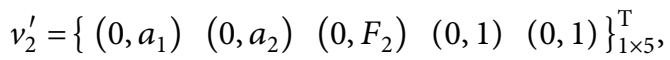

$$
\begin{aligned}
& B_{1}=\left(\begin{array}{lllll}
2 & 0 & 0 & 0 & 0 \\
0 & 0 & 0 & 0 & 0 \\
0 & 0 & 0 & 0 & 0 \\
0 & 0 & 0 & 1 & 0 \\
0 & 0 & 0 & 0 & 0
\end{array}\right), \\
& B_{2}=\left(\begin{array}{lllll}
0 & 0 & 0 & 0 & 0 \\
0 & 0 & 0 & 0 & 0 \\
0 & 0 & 0 & 0 & 0 \\
0 & 0 & 0 & 0 & 0 \\
0 & 0 & 0 & 0 & 0
\end{array}\right),
\end{aligned}
$$

$$
\begin{aligned}
& \text { Step 5. Proving of the universal unfolding of the normal } \\
& \text { form of } h \text {. }
\end{aligned}
$$

Proposition 7. The codimension of $h$ in $\overrightarrow{\mathrm{E}}_{z, \lambda}$ is 3 , and the universal unfolding of (39) is given as

$$
G=\left(a_{1}^{2}+2 \varepsilon_{1} \sigma_{1} a_{2}+2 \varepsilon_{3} F_{2}+\tau_{1}, a_{2}^{2}-F_{2}^{2}+2 \varepsilon_{2} F_{2} a_{1}+\tau_{2}\right),
$$

where $\varepsilon_{i}(i=1,2,3)$ is a sign function.

Proof. According to Theorem 2.1 and equation 2.7 (page 211 in Golubitsky and Schaeffer II, 1985), it can be obtained that

$$
\begin{aligned}
T(h, 1)= & \left(M^{2}+M\left\langle F_{2}\right\rangle+\left\langle\sigma_{1}, F_{2}^{2}\right\rangle+\left\langle\tau_{1}\right\rangle+\left\langle\tau_{2}\right\rangle\right) \vec{E}_{z, \lambda} \\
& +R\left\{\left(a_{1}, 0\right),\left(0, a_{2}\right),\left(0, F_{2}\right),(1,0),(0,1)\right\} .
\end{aligned}
$$


It is not difficult to see that the largest intrinsic ideal $\operatorname{ItrT}(h, 1)$ contained in $\mathrm{T}(h, 1)$ is just

$$
\begin{aligned}
\operatorname{ItrT}(h, 1) & =\left(\mathrm{M}^{2}+\mathrm{M}\left\langle\sigma_{1}, F_{2}\right\rangle+\left\langle F_{2}^{2}\right\rangle+\left\langle\tau_{1}\right\rangle+\left\langle\tau_{2}\right\rangle\right) \overrightarrow{\mathrm{E}}_{z, \lambda}, \\
{[\operatorname{ItrT}(h, 1)]^{\perp} } & =\mathrm{R}\left\{\left(a_{1}, 0\right),\left(a_{2}, 0\right),\left(\sigma_{1}, 0\right),\left(F_{2}, 0\right),\left(0, a_{1}\right),\left(0, a_{2}\right),\left(0, F_{2}\right),\left(0, \sigma_{1}\right),(1,0),(0,1)\right\},
\end{aligned}
$$

whose dimension is 10 .

Clearly, the dimension of $\mathrm{R}\left\{\left(a_{1}, 0\right),\left(0, a_{2}\right),\left(0, F_{2}\right)\right.$, $(1,0),(0,1)\}$ is 5 ; hence, there exists a basis for a subspace of $[\operatorname{ItrT}(h, 1)]^{\perp}$, which is complementary to $\mathrm{R}\left\{\left(a_{1}, 0\right)\right.$, $\left.\left(0, a_{2}\right),\left(0, F_{2}\right),(1,0),(0,1)\right\}$.

Consequently, it is easy to find a complementary subspace to $\mathrm{T}(h, 1)$, spanned by

$$
\begin{aligned}
& \left(a_{2}, 0\right), \\
& \left(\sigma_{1}, 0\right), \\
& \left(F_{2}, 0\right), \\
& \left(0, a_{1}\right), \\
& \left(0, \sigma_{1}\right) .
\end{aligned}
$$

According to Proposition 5, in (64), for $\left(a_{2}, 0\right)$ and $\left(0, a_{1}\right)$, there exist two sign functions $\varepsilon_{1}$ and $\varepsilon_{2}$, such that $\left(\varepsilon_{1} \sigma_{1} a_{2}, 0\right)$ and $\left(0, \varepsilon_{2} F_{2} a_{1}\right)$ will exist in universal unfolding of $h$. For $\left(F_{2}, 0\right)$, there exist one sign function $\varepsilon_{3}$, such that $\left(\varepsilon_{3} F_{2}, 0\right)$ will exist in universal unfolding of $h$. For $\left(\sigma_{1}, 0\right)$ and $\left(0, \sigma_{1}\right)$, there exists one sign function $\varepsilon_{3}$, such that $\left(\varepsilon_{1} F_{2}, 0\right)$ will exist in universal unfolding of $h$.

According to the above analysis, the universal unfolding of $h$ is given by

$$
G=\left(a_{1}^{2}+2 \varepsilon_{1} \sigma_{1} a_{2}+2 \varepsilon_{3} F_{2}+\tau_{1}, a_{2}^{2}-F_{2}^{2}+2 \varepsilon_{2} F_{2} a_{1}+\tau_{2}\right) .
$$
that

Using Proposition 5, when $\varepsilon_{i}=0$, (65) can be obtained

$$
G\left(a_{1}, a_{2}, \sigma_{1}, F_{2}, \tau_{1}, \tau_{2}\right)=\left(a_{1}^{2}+\tau_{1}, a_{2}^{2}-F_{2}^{2}+\tau_{2}\right)
$$

When $\varepsilon_{i} \neq 0$, it is obtained from (65) that

$$
\operatorname{det}\left(\begin{array}{cccccc}
0 & 2 & 0 & 0 & 0 & 0 \\
0 & 0 & 0 & 0 & 0 & 2 \\
0 & 0 & 2 \varepsilon_{1} & 0 & 0 & 0 \\
2 \varepsilon_{3} & 0 & 0 & 0 & 2 \varepsilon_{2} & 0 \\
0 & 0 & 0 & 1 & 0 & 0 \\
1 & 0 & 0 & 0 & 0 & 0
\end{array}\right) \neq 0 .
$$

Obviously, Proposition 1 and Proposition 7 are the same.

\section{Conclusions}

The bifurcation of the laminated piezoelectric composite rectangular plate structure with $1: 2$ internal resonance is a high codimensional problem. The universal unfolding with codimension 3 of the bifurcation equations is carried out using the extended singularity theory method. Furthermore, the main material parameters $\alpha_{6}, \alpha_{7}$, and $\beta_{7}$ that affect the dynamic behavior of the laminated piezoelectric composite rectangular plate structure near the singularity under transverse excitation are revealed by the transition set of universal unfolding with codimension 3 .

The transition set divides the parameters space into three kinds of different persistent regions, the results indicate that entering region (1) from region (2), jumping will occur, the hysteresis will occur when entering region (2) from region (3), and the composite laminated piezoelectric rectangular plate structure subjected to small perturbations is in unstable state. The numerical results from the above analysis show that the stability of the proposed system is better when $\kappa<0$. It can pick a set of appropriate initial value for parameters $\alpha_{6}, \alpha_{7}$, and $\beta_{7}$, such that period ratio $\kappa$ is chosen in region (1) or (2); thus, the system is stable and the motion is periodic.

For the composite laminated piezoelectric rectangular plate structure, it is demonstrated that there exists abundant dynamic bifurcation patterns in a small perturbation sense. Some new chaotic dynamics are also presented. Clearly, these results provide some inspiration and guidance for the analysis and dynamic designs of this structure.

\section{Appendix}

The coefficients of equation (15) (Zhang and Yao, 2009) are presented as follows: 


$$
\begin{aligned}
& k_{1}=\frac{2688}{715} \frac{\alpha A_{12}}{\beta^{2} \pi A_{11}+\alpha^{2} \pi A_{66}}+\frac{27264}{715} \frac{\beta^{2} A_{11}}{\alpha \beta^{2} \pi A_{11}+\alpha^{3} \pi A_{66}}-\frac{384}{715} \frac{\alpha A_{66}}{\beta^{2} \pi A_{11}+\alpha^{2} \pi A_{66}}, \\
& k_{2}=\frac{2688}{715} \frac{\alpha A_{12}}{\beta^{2} \pi A_{11}+\alpha^{2} \pi A_{66}}+\frac{27264}{715} \frac{\beta^{2} A_{11}}{\alpha \beta^{2} \pi A_{11}+\alpha^{3} \pi A_{66}}-\frac{384}{715} \frac{\alpha A_{66}}{\beta^{2} \pi A_{11}+\alpha^{2} \pi A_{66}} \text {, } \\
& k_{3}=\frac{3328}{33075} \frac{\alpha A_{12}}{\beta^{2} \pi A_{11}+\alpha^{2} \pi A_{66}}+\frac{3328}{33075} \frac{\beta^{2} A_{11}}{\alpha \beta^{2} \pi A_{11}+\alpha^{3} \pi A_{66}}-\frac{10496}{3696} \frac{\alpha A_{66}}{\beta^{2} \pi A_{11}+\alpha^{2} \pi A_{66}} \text {, } \\
& k_{4}=\frac{896}{225} \frac{\alpha A_{12}}{9 \beta^{2} \pi A_{11}+\alpha^{2} \pi A_{66}}+\frac{896}{225} \frac{\beta^{2} A_{11}}{9 \alpha \beta^{2} \pi A_{11}+\alpha^{3} \pi A_{66}}-\frac{128}{225} \frac{\alpha A_{66}}{9 \beta^{2} \pi A_{11}+\alpha^{2} \pi A_{66}} \text {, } \\
& k_{5}=\frac{81792}{1001} \frac{\alpha A_{12}}{9 \beta^{2} \pi A_{11}+\alpha^{2} \pi A_{66}}+\frac{1152}{1001} \frac{\beta^{2} A_{11}}{9 \alpha \beta^{2} \pi A_{11}+\alpha^{3} \pi A_{66}}-\frac{1152}{1001} \frac{\alpha A_{66}}{9 \beta^{2} \pi A_{11}+\alpha^{2} \pi A_{66}} \text {, } \\
& k_{6}=\frac{264448}{40425} \frac{\alpha A_{66}}{9 \beta^{2} \pi A_{11}+\alpha^{2} \pi A_{66}}+\frac{23808}{13475} \frac{\beta^{2} A_{11}}{9 \alpha \beta^{2} \pi A_{11}+\alpha^{3} \pi A_{66}}+\frac{29952}{13475} \frac{\alpha A_{12}}{9 \beta^{2} \pi A_{11}+\alpha^{2} \pi A_{66}} \text {, } \\
& k_{7}=\frac{27264}{715} \frac{\beta A_{12}}{\alpha^{2} \pi A_{22}+\beta^{2} \pi A_{66}}+\frac{2688}{715} \frac{\alpha^{2} A_{22}}{\alpha^{2} \beta \pi A_{22}+\beta^{3} \pi A_{66}}-\frac{384}{715} \frac{\beta A_{66}}{\alpha^{2} \pi A_{22}+\beta^{2} \pi A_{66}} \text {, } \\
& k_{8}=\frac{2688}{715} \frac{\beta A_{12}}{\alpha^{2} \pi A_{22}+\beta^{2} \pi A_{66}}+\frac{27264}{715} \frac{\alpha^{2} A_{22}}{\alpha^{2} \beta \pi A_{22}+\beta^{3} \pi A_{66}}-\frac{384}{715} \frac{\beta A_{66}}{\alpha^{2} \pi A_{22}+\beta^{2} \pi A_{66}} \text {, } \\
& k_{9}=-\frac{3328}{33075} \frac{\beta A_{12}}{\alpha^{2} \pi A_{22}+\beta^{2} \pi A_{66}}+\frac{3328}{33075} \frac{\alpha^{2} A_{22}}{\alpha^{2} \beta \pi A_{22}+\beta^{3} \pi A_{66}}-\frac{10496}{3369} \frac{\beta A_{66}}{\alpha^{2} \pi A_{22}+\beta^{2} \pi A_{66}}, \\
& k_{10}=\frac{81792}{1001} \frac{\beta A_{12}}{9 \alpha^{2} \pi A_{22}+\beta^{2} \pi A_{66}}-\frac{1152}{1001} \frac{\alpha^{2} A_{22}}{9 \alpha^{2} \pi A_{22}+\beta^{3} \pi A_{66}}-\frac{1152}{1001} \frac{\beta A_{66}}{9 \alpha^{2} \pi A_{22}+\beta^{2} \pi A_{66}}, \\
& k_{11}=\frac{896}{225} \frac{\beta A_{12}}{9 \alpha^{2} \pi A_{22}+\beta^{2} \pi A_{66}}-\frac{896}{225} \frac{\alpha^{2} A_{22}}{9 \alpha^{2} \pi A_{22}+\beta^{3} \pi A_{66}}-\frac{128}{225} \frac{\beta A_{66}}{9 \alpha^{2} \pi A_{22}+\beta^{2} \pi A_{66}}, \\
& k_{12}=\frac{29952}{13475} \frac{\beta A_{12}}{9 \alpha^{2} \pi A_{22}+\beta^{2} \pi A_{66}}-\frac{23808}{13475} \frac{\alpha^{2} A_{22}}{9 \alpha^{2} \pi A_{22}+\beta^{3} \pi A_{66}}-\frac{264448}{40425} \frac{\beta A_{66}}{9 \alpha^{2} \pi A_{22}+\beta^{2} \pi A_{66}},
\end{aligned}
$$

in particular, for arbitrary constants $k_{i}(i=13, \ldots, 18)$, the expressions 


$$
\begin{aligned}
u_{0}= & u_{1}(t) \cos \frac{\pi x}{2 \alpha} \cos \frac{\pi y}{2 \beta}+u_{2}(t) \cos \frac{3 \pi x}{2 \alpha} \cos \frac{\pi y}{2 \beta} \\
& +k_{13} x+k_{14} y+k_{15}, \\
v_{0}= & v_{1}(t) \cos \frac{\pi y}{2 \beta} \cos \frac{\pi x}{2 \alpha}+v_{2}(t) \cos \frac{3 \pi y}{2 \beta} \cos \frac{\pi x}{2 \alpha} \\
& +k_{16} x+k_{17} y+k_{18},
\end{aligned}
$$

are also the solutions of equation (12) (Zhang and Yao, 2009).

The coefficients of equation (1) in this work are presented as follows:

$$
\begin{aligned}
& m_{1}=I_{0}-\frac{J_{4} k_{7}}{\alpha \pi c_{1}}+\frac{I_{6}}{\beta^{2} \pi^{2} c_{1}^{2}}+\frac{I_{6}}{\alpha^{2} \pi^{2} c_{1}^{2}}-\frac{J_{4} k_{9}}{\beta \pi c_{1}} \\
& m_{2}=I_{0}-\frac{3 J_{4} k_{8}}{\alpha \pi c_{1}}+\frac{9 I_{6}}{\alpha^{2} \pi^{2} c_{1}^{2}}+\frac{I_{6}}{\beta^{2} \pi^{2} c_{1}^{2}}-\frac{J_{4} k_{10}}{\beta \pi c_{1}} \\
& \omega_{1}^{2}=\pi^{2} \frac{F_{44} c_{2}^{2}-2 D_{44} c_{2}+A_{44}}{m_{1} \beta^{2}}+\pi^{2} \frac{-2 D_{55} c_{2}+A_{55}+F_{55} c_{2}^{2}}{m_{1} \alpha^{2}}+\pi \frac{A_{55} k_{7}+F_{55} c_{2}^{2} k_{7}-2 D_{55} c_{2} k_{7}}{m_{1} \alpha} \\
& +\pi \frac{A_{44} k_{9}+F_{44} c_{2}^{2} k_{9}-2 D_{44} c_{2} k_{9}}{m_{1} \beta}+\pi^{3} \frac{-2 c_{1} F_{11} k_{7}+H_{11} c_{1}^{2} k_{7}}{m_{1} \alpha^{3}}+\pi^{4} \frac{H_{22} c_{1}^{2}}{m_{1} \beta^{4}}+\pi^{3} \frac{H_{22} c_{1}^{2} k_{9}-c_{1} F_{22} k_{9}}{m_{1} \beta^{3}} \\
& +\pi \frac{{ }_{4}^{4} H_{11} c_{1}^{2}}{m_{1} \alpha^{4}}+\pi \frac{{ }_{4}^{4} H_{12} c_{1}^{2}+4 H_{66} c_{1}^{2}+H_{21} c_{1}^{2}}{m_{1} \alpha^{2} \beta^{2}} \\
& +\pi \frac{{ }_{3} H_{12} c_{1}^{2} k_{9}-c_{1} F_{12} k_{9}-2 c_{1} F_{66} k_{9}+2 H_{66} c_{1}^{2} k_{9}}{m_{1} \alpha^{2} \beta}+\pi^{3} \frac{{ }_{21} c_{1}^{2} k_{7}-c_{1} F_{21} k_{7}-2 c_{1} F_{66} k_{7}+2 H_{66} c_{1}^{2} k_{7}}{m_{1} \beta^{2} \alpha}, \\
& \omega_{2}^{2}=\pi \frac{A_{44} k_{10}+F_{44} c_{2}^{2} k_{10}-2 D_{44} c_{2} k_{10}}{m_{2} \beta}+\pi^{2} \frac{F_{44} c_{2}^{2}-2 D_{44} c_{2}+A_{44}}{m_{2} \beta^{2}}+\pi^{2} \frac{-18 D_{55} c_{2}+9 A_{55}+9 F_{55} c_{2}^{2}}{m_{2} \alpha^{2}} \\
& +\pi \frac{3 A_{55} k_{8}+3 F_{55} c_{2}^{2} k_{8}-6 D_{55} c_{2} k_{8}}{m_{2} \alpha}+\pi^{3} \frac{-c_{1} F_{22} k_{10}+H_{22} c_{1}^{2} k_{10}}{m_{2} \beta^{3}}+\pi^{4} \frac{H_{22} c_{1}^{2}}{m_{2} \beta^{4}} \\
& +\pi^{3} \frac{27 H_{11} c_{1}^{2} k_{8}-27 c_{1} F_{11} k_{8}}{m_{2} \alpha^{3}}+\pi^{4} \frac{81 H_{11} c_{1}^{2}}{m_{2} \alpha^{4}}+\pi^{4} \frac{36 H_{66} c_{1}^{2}+9 H_{12} c_{1}^{2}+9 H_{21} c_{1}^{2}}{m_{2} \alpha^{2} \beta^{2}} \\
& +\pi \frac{3 H_{21} c_{1}^{2} k_{8}-3 c_{1} F_{21} k_{8}-6 c_{1} F_{66} k_{8}+6 H_{66} c_{1}^{2} k_{8}}{m_{2} \beta^{2} \alpha}+\pi^{3} \frac{9 H_{12} c_{1}^{2} k_{10}-9 c_{1} F_{12} k_{10}-18 c_{1} F_{66} k_{10}+18 H_{66} c_{1}^{2} k_{10}}{m_{2} \alpha^{2} \beta}, \\
& \alpha_{2}=\frac{\pi^{2} q_{x}}{\alpha^{2}} \\
& \alpha_{3}=\frac{\pi^{2} q_{y}}{\beta^{2}} \\
& \alpha_{4}=-\frac{\pi^{2} N_{x}^{p}}{\alpha^{2}}-\frac{\pi^{2} N_{y}^{p}}{\beta^{2}}
\end{aligned}
$$




$$
\begin{aligned}
& \alpha_{5}=-\frac{208 \pi A_{11} k_{1}}{315 \alpha^{3}}+\frac{112 \pi A_{22} k_{6}}{45 \beta^{3}}+\frac{64 \pi A_{66} k_{1}}{945 \alpha \beta^{2}}+\frac{64 \pi A_{66} k_{4}}{225 \alpha^{2} \beta}+\frac{64 \pi A_{21} k_{1}}{45 \alpha \beta^{2}} \\
& +\frac{\pi^{4} A_{12}}{32 \alpha^{2} \beta^{2}}-\frac{112 \pi A_{22} k_{4}}{225 \beta^{3}}+\frac{64 \pi A_{21} k_{1}}{945 \alpha \beta^{2}}+\frac{5 \pi^{4} A_{21}}{32 \alpha^{2} \beta^{2}}+\frac{64 \pi A_{12} k_{6}}{45 \alpha^{2} \beta}+\frac{64 \pi A_{66} k_{3}}{45 \alpha \beta^{2}} \\
& +\frac{3 \pi^{4} A_{66}}{8 \alpha^{2} \beta^{2}}-\frac{64 \pi A_{12} k_{4}}{25 \alpha^{2} \beta}-\frac{64 \pi A_{66} k_{6}}{45 \alpha^{2} \beta}-\frac{27 \pi^{4} A_{11}}{32 \alpha^{4}}+\frac{9 \pi^{4} A_{22}}{32 \beta^{4}}+\frac{112 \pi A_{11} k_{3}}{45 \beta^{3}}, \\
& \alpha_{6}=\frac{64 \pi A_{66} k_{3}}{945 \alpha \beta^{2}}-\frac{64 \pi A_{21} k_{3}}{945 \alpha \beta^{2}}-\frac{208 \pi A_{11} k_{3}}{315 \alpha^{3}}+\frac{64 \pi A_{66} k_{6}}{225 \alpha^{2} \beta}+\frac{64 \pi A_{21} k_{2}}{45 \alpha \beta^{2}}+\frac{112 \pi A_{22} k_{5}}{45 \beta^{3}} \\
& -\frac{9 \pi^{4} A_{22}}{16 \beta^{4}}-\frac{\pi^{4} A_{12}}{16 \alpha \beta^{2}}-\frac{64 \pi A_{66} k_{5}}{45 \alpha^{2} \beta}-\frac{64 \pi A_{66} k_{2}}{45 \alpha \beta^{2}}-\frac{112 \pi A_{22} k_{6}}{225 \beta^{3}}-\frac{81 \pi^{4} A_{11}}{16 \alpha^{4}}+\frac{112 \pi A_{11} k_{2}}{45 \alpha^{3}} \\
& +\frac{64 \pi A_{12} k_{5}}{45 \alpha^{2} \beta}-\frac{208 \pi A_{12} k_{6}}{25 \alpha^{2} \beta}-\frac{9 \pi^{4} A_{21}}{16 \alpha^{2} \beta^{2}} \\
& \alpha_{7}=\frac{64 \pi A_{21} k_{1}}{45 \alpha \beta^{2}}-\frac{64 \pi A_{66} k_{4}}{45 \alpha^{2} \beta}+\frac{64 \pi A_{12} k_{4}}{45 \alpha^{2} \beta}-\frac{\pi^{4} A_{12}}{32 \alpha^{2} \beta^{2}} \\
& +\frac{112 \pi A_{11}}{45 \alpha \beta^{2}}-\frac{\pi^{4} A_{21}}{32 \alpha^{2} \beta^{2}}-\frac{9 \pi^{4} A_{11}}{32 \alpha^{4}}-\frac{\pi A_{66} k_{1}}{45 \alpha \beta^{2}}-\frac{\pi A_{66}}{8 \alpha^{2} \beta^{2}}+\frac{112 \pi A_{22} k_{4}}{45 \beta^{3}}-\frac{9 \pi^{4} A_{22}}{32 \beta^{4}}, \\
& \alpha_{8}=\frac{64 \pi A_{21} k_{2}}{945 \alpha \beta^{2}}-\frac{208 \pi A_{11} k_{2}}{315 \alpha^{3}}+\frac{64 \pi A_{66} k_{2}}{945 \alpha \beta^{2}}-\frac{\pi A_{22} k_{5}}{225 \beta^{3}}-\frac{64 \pi A_{12} k_{5}}{25 \alpha^{2} \beta}+\frac{64 \pi A_{66} k_{5}}{225 \alpha^{2} \beta} \\
& F_{1}=-\frac{16 q_{3}}{3 \pi^{2}} \\
& \beta_{2}=-\frac{9 \pi^{2} q_{x}}{\alpha^{2}} \\
& \beta_{3}=-\frac{\pi^{2} q_{y}}{\beta^{2}} \\
& \beta_{4}=\frac{9 \pi^{2} N_{x}^{p}}{\alpha^{2}}+\frac{\pi^{2} N_{y}^{p}}{\beta^{2}} \\
& \beta_{5}=-\frac{192 \pi A_{66} k_{3}}{143 \alpha \beta^{2}}+\frac{264 \pi A_{12} k_{6}}{175 \alpha^{2} \beta}-\frac{192 \pi A_{66} k_{6}}{175 \alpha^{2} \beta}+\frac{48 \pi A_{22} k_{6}}{25 \beta^{3}}+\frac{192 \pi A_{21} k_{3}}{143 \alpha \beta^{2}}-\frac{64 \pi A_{21} k_{2}}{945 \alpha \beta^{2}}, \\
& \beta_{6}=-\frac{192 \pi A_{66} k_{4}}{143 \alpha \beta^{2}}+\frac{264 \pi A_{12} k_{6}}{175 \alpha^{2} \beta}-\frac{192 \pi A_{66} k_{6}}{175 \alpha^{2} \beta}+\frac{48 \pi A_{22} k_{6}}{25 \beta^{3}}+\frac{192 \pi A_{21} k_{4}}{143 \alpha \beta^{2}}-\frac{64 \pi A_{21} k_{1}}{945 \alpha \beta^{2}} \text {, } \\
& \beta_{7}=\frac{192 \pi\left(-A_{66} k_{2}+A_{21} k_{2}\right)}{143 \alpha \beta^{2}}+\frac{48 \pi A_{22} k_{5}}{25 \beta^{3}}-\frac{729 \pi^{4} A_{11}}{32 \alpha^{4}}-\frac{9 \pi^{4} A_{22}}{32 \beta^{4}}+\frac{9 \pi^{4}\left(-4 A_{66}-A_{21}-A_{12}\right)}{32 \alpha^{2} \beta^{2}} \\
& +\frac{408 \pi A_{11} k_{2}}{143 \alpha^{3}}+\frac{\pi\left(264 A_{12} k_{5}-192 A_{66} k_{5}\right)}{175 \alpha^{2} \beta}, \\
& \beta_{8}=\frac{64 \pi\left(-A_{21} k_{1}+A_{66} k_{1}\right)}{945 \alpha \beta^{2}}-\frac{112 \pi A_{22} k_{4}}{225 \beta^{3}}-\frac{9 \pi^{4} A_{11}}{32 \alpha^{4}}+\frac{3 \pi^{4} A_{22}}{32 \beta^{4}}+\frac{\pi^{4}\left(4 A_{66}-A_{21}+3 A_{12}\right)}{32 \alpha^{2} \beta^{2}} \\
& -\frac{208 \pi A_{11} k_{1}}{315 \alpha^{3}}+\frac{64 \pi\left(-9 A_{12} k_{4}+A_{66} k_{4}\right)}{\alpha^{2} \beta}, \\
& F_{2}=-\frac{16 q_{3}}{3 \pi^{2}}
\end{aligned}
$$




\section{Data Availability}

The data used to support the findings of the study are included within the article.

\section{Conflicts of Interest}

The authors declare that they have no conflicts of interest.

\section{Acknowledgments}

The authors gratefully acknowledge the support of the National Natural Science Foundation of China (NNSFC) (11832002) and the fund of Independent Research and Development Program of China Aerospace Science and Technology (503Y-Y-Y-FZLDTX-20).

\section{References}

[1] D. G. Schaeffer and M. A. Golubitsky, "Bifurcation analysis near a double eigenvalue of a model chemical reaction," Archive for Rational Mechanics and Analysis, vol. 75, no. 4, pp. 315-347, 1981.

[2] J. Martinet, Singularities of Smooth Functions and Maps, Cambridge University Press, Cambridge, UK, 1982.

[3] Y. S. Chen and W. F. Langford, "“The subharmonic bifurcation solution of nonlinear Mathieu's equation and Eurler dynamically buckling problem," Acta Mechanica Sinica, vol. 4, no. 4, pp. 350-362, 1988.

[4] J. D. Jin and G. S. Zou, "Bifurcations and chaotic motions in the autonomous system of a restrained pipe conveying fluidfluid," Journal of Sound and Vibration, vol. 260, no. 5, pp. 783-805, 2003.

[5] F. Chen, J. Liang, Y. Chen, X. Liu, and H. Ma, "Bifurcation analysis of an arch structure with parametric and forced excitation," Mechanics Research Communications, vol. 34, no. 3, pp. 213-221, 2007.

[6] O. Nigol and P. Buchan, "Conductor galloping part I - den hartog mechanism," IEEE Transactions on Power Apparatus and Systems, vol. 100, no. 2, pp. 699-707, 1981.

[7] O. Nigol and P. Buchan, "Conductor galloping-part II torsional mechanism," IEEE Transactions on Power Apparatus and Systems, vol. 100, no. 2, pp. 708-720, 1981.

[8] P. Yu, N. Popplewell, and A. H. Shah, "Instability trends of inertially coupled galloping Part II: periodic vibrations," Journal of Sound and Vibration, vol. 183, no. 4, pp. 679-691, 1995.

[9] J. E. Futer, A. M. Sitta, and I. Stewart, "Singularity theory and equivariant bifurcation problems with parameter symmetry," Mathematical Proceedings of the Cambridge Philosophical Society, vol. 120, no. 3, pp. 547-578, 1996.

[10] A. M. Sitta, "Singularity Theory and Equivariant bifurcation problems with parameter symmetry," Ph. D. thesis, University of Warwick and USP-sao Carlos, São Carlos, Brazil, 1993.

[11] A. H. Lari-Lavassani and Y.-C. Lu, "Equivariant multiparameter bifurcation via singularity theory," Journal of $D y$ namics and Differential Equations, vol. 5, no. 2, pp. 189-218, 1993.

[12] A. P. Seyranian and A. A. Mailybaev, Multiparameter Stability Theory with Mechanical Application, World Scientific, Singapore, 2003.
[13] Z. Qin and Y. Chen, "Singular analysis of bifurcation systems with two parameters," Acta Mechanica Sinica, vol. 26, no. 3, pp. 501-507, 2010.

[14] D. M. Zhang and F. Q. Chen, "Stability and bifurcation for a simply supported functionally graded material plate with oneto-one internal resonance," Journal of Applied Mathematics, vol. 2014, Article ID 414903, , 2014.

[15] Y. H. Guo and W. Zhang, "Bifurcation analysis of composite laminated piezoelectric rectangular plate structure in the case of 1:2 internal resonance," Mathematical Problems in Engineering, vol. 2019, Article ID 6830274, , 2019.

[16] T. R. Tauchert, F. Ashida, N. Noda, S. Adali, and V. Verijenko, "Developments in thermopiezoelasticity with relevance to smart composite structures," Composite Structures, vol. 48, no. 1-3, pp. 31-38, 2000.

[17] D. Halim and S. O. Reza Moheimani, "An optimization approach to optimal placement of collocated piezoelectric actuators and sensors on a thin plate," Mechatronics, vol. 13, no. 1, pp. 27-47, 2003.

[18] W. Zhang, C. Z. Song, and M. Ye, "Further studies on nonlinear oscillations and chaos of a rectangular symmetric cross-by laminated plate under parametric excitation," International Journal of Bifurcation and Chaos, vol. 16, no. 2, pp. 325-347, 2006.

[19] C. N. Della and D. Shu, "Vibration of delaminated composite laminates: a review," Applied Mechanics Reviews, vol. 60, no. 1, pp. 1-20, 2007.

[20] Y. X. Hao, L. H. Chen, and W. Zhang, "Nonlinear oscillations, bifurcations and chaos of functionally graded materials plate," Journal of Sound and Vibration, vol. 312, no. 4, pp. 862-892, 2008.

[21] V. G. Karnaukhov and V. Tkachenko Ya, "Damping the vibrations of a rectangular plate with piezoelectric actuators," International Applied Mechanics, vol. 44, no. 1, pp. 78-84, 2008.

[22] Y. Liu, Z. Qin, and F. Chu, "Nonlinear forced vibrations of FGM sandwich cylindrical shells with porosities on an elastic substrate," Nonlinear Dynamics, vol. 104, no. 2, pp. 1007-1021, 2021.

[23] Y. F. Liu, Z. Y. Qin, and F. L. Chu, "Nonlinear forced vibrations of functionally graded piezoelectric cylindrical shells under electric-thermo-mechanical loads," International Journal of Mechanical Sciences, vol. 2021, Article ID 106474, 2021.

[24] W. Zhang, Z. Yao, and M. Yao, "Periodic and chaotic dynamics of composite laminated piezoelectric rectangular plate with one-to-two internal resonance," Science in China Series E: Technological Sciences, vol. 52, no. 3, pp. 731-742, 2009.

[25] M. Golubitsky and D. G. Schaeffer, "Singularities and groups in bifurcation theory," in Applied Mathematical SciencesSpringer, New York, NY, USA, 1985. 